\title{
Distinct extracellular-matrix remodeling events precede symptoms of inflammation
}

Shimshoni, Elee; Adir, Idan; Afik, Ran; Solomonov, Inna; Shenoy, Anjana; Adler, Miri; Puricelli, Luca; Sabino, Fabio Mira Rocha; Savickas, Simonas; Mouhadeb, Odelia

Total number of authors:

22

Published in:

Matrix Biology

Link to article, DOI:

10.1016/j.matbio.2020.11.001

Publication date:

2021

Document Version

Peer reviewed version

Link back to DTU Orbit

Citation (APA):

Shimshoni, E., Adir, I., Afik, R., Solomonov, I., Shenoy, A., Adler, M., Puricelli, L., Sabino, F. M. R., Savickas, S., Mouhadeb, O., Gluck, N., Fishman, S., Werner, L., Salame, T-M., Shouval, D. S., Varol, C., auf dem Keller, U., Podestà, A., Geiger, T., ... Sagi, I. (2021). Distinct extracellular-matrix remodeling events precede symptoms of inflammation. Matrix Biology, 96, 47-68. https://doi.org/10.1016/j.matbio.2020.11.001

\section{General rights}

Copyright and moral rights for the publications made accessible in the public portal are retained by the authors and/or other copyright owners and it is a condition of accessing publications that users recognise and abide by the legal requirements associated with these rights.

- Users may download and print one copy of any publication from the public portal for the purpose of private study or research.

- You may not further distribute the material or use it for any profit-making activity or commercial gain

- You may freely distribute the URL identifying the publication in the public portal 


\section{Journal Pre-proof}

Distinct extracellular-matrix remodeling events precede symptoms of inflammation

Elee Shimshoni, Idan Adir, Ran Afik, Inna Solomonov, Anjana Shenoy, Miri Adler, Luca Puricelli , Fabio Sabino, Simonas Savickas, Odelia Mouhadeb , Nathan Gluck , Sigal Fishman, Lael Werner, Tomer-Meir Salame, Dror S. Shouval, Chen Varol, Ulrich auf dem Keller, Alessandro Podestà, Tamar Geiger, Paolo Milani, Uri Alon , Irit Sagi

PII:

DOI: S0945-053X(20)30103-7

Reference: https://doi.org/10.1016/j.matbio.2020.11.001 MATBIO 1658

To appear in:

Matrix Biology

Received date:

27 March 2020

Revised date:

15 November 2020

Accepted date:

17 November 2020

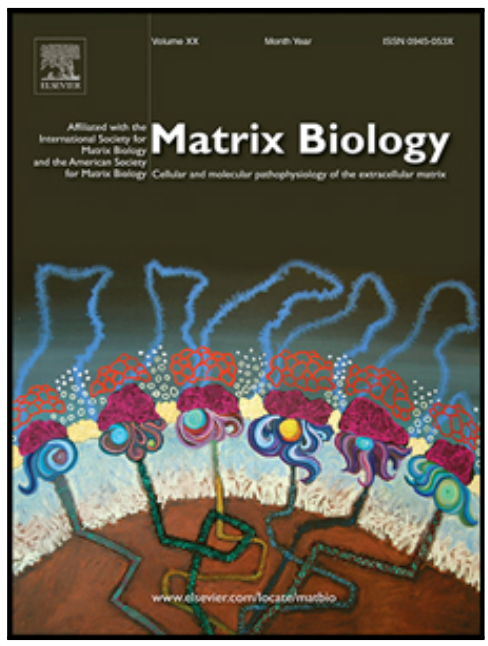

Please cite this article as: Elee Shimshoni, Idan Adir, Ran Afik, Inna Solomonov, Anjana Shenoy, Miri Adler, Luca Puricelli, Fabio Sabino, Simonas Savickas, Odelia Mouhadeb, Nathan Gluck, Sigal Fishman, Lael Werner, Tomer-Meir Salame, Dror S. Shouval, Chen Varol, Ulrich auf dem Keller, Alessandro Podestà , Tamar Geiger, Paolo Milani, Uri Alon, Irit Sagi, Distinct extracellular-matrix remodeling events precede symptoms of inflammation, Matrix Biology (2020), doi: https://doi.org/10.1016/j.matbio.2020.11.001

This is a PDF file of an article that has undergone enhancements after acceptance, such as the addition of a cover page and metadata, and formatting for readability, but it is not yet the definitive version of record. This version will undergo additional copyediting, typesetting and review before it is published in its final form, but we are providing this version to give early visibility of the article. Please note that, during the production process, errors may be discovered which could affect the content, and all legal disclaimers that apply to the journal pertain.

(C) 2020 Elsevier B.V. All rights reserved. 


\section{Highlights}

- Investigating extracellular-matrix (ECM) dynamics during inflammatory disease onset reveals a unique ECM state, which we term the "pre-symptomatic ECM".

- The pre-symptomatic ECM is characterized by distinct structural, mechanical and compositional characteristics.

- Both subclinical infiltration of immune cells bearing remodeling enzymes and elevated gelatinase activity in the epithelium contribute to the pre-symptomatic ECM. 


\title{
Distinct extracellular-matrix remodeling events precede symptoms of inflammation
}

Authors: Elee Shimshoni ${ }^{1 \dagger}$, Idan Adir $^{1}$, Ran Afik ${ }^{1}$, Inna Solomonov ${ }^{1}$, Anjana Shenoy ${ }^{2}$, Miri Adler $^{3}$, Luca Puricelli ${ }^{4}$, Fabio Sabino ${ }^{5}$, Simonas Savickas ${ }^{5}$, Odelia Mouhadeb ${ }^{6,8}$, Nathan Gluck ${ }^{6,8}$, Sigal Fishman ${ }^{6,8}$, Lael Werner ${ }^{7,8}$, Tomer-Meir Salame ${ }^{9}$, Dror S. Shouval ${ }^{7,8}$, Chen Varol ${ }^{6,8}$, Ulrich auf dem Keller ${ }^{5}$, Alessandro Podestà ${ }^{4}$, Tamar Geiger ${ }^{2}$, Paolo Milani ${ }^{4}$, Uri Alon ${ }^{3}$ and Irit Sagi ${ }^{*}$

\author{
Affiliations: \\ ${ }^{1}$ Department of Biological Regulation, Weizmann Institute of Science, Rehovot, Israel \\ ${ }^{2}$ Department of Human Molecular Genetics and Biochemistry, Sackler Faculty of Medicine, Tel \\ Aviv University, Tel Aviv-Yafo, Israel \\ ${ }^{3}$ Department of Molecular Cell Biology, Weizmann Institute of Science, Rehovot, Israel \\ ${ }^{4}$ CIMAINA and Department of Physics, Università degli Studi di Milano, Milano, Italy \\ ${ }^{5}$ Department of Biotechnology and Biomedicine, Technical University of Denmark, Kgs. \\ Lyngby, Denmark \\ ${ }^{6}$ Research Center for Digestive Tract and Liver Diseases, Tel Aviv Sourasky Medical Center, Tel \\ Aviv-Yafo, Israel \\ ${ }^{7}$ Pediatric Gastroenterology Unit, Edmond and Lily Safra Children's Hospital, Sheba Medical \\ Center, Tel Hashomer, Israel \\ ${ }^{8}$ Sackler Faculty of Medicine, Tel Aviv University, Tel Aviv-Yafo, Israel \\ ${ }^{9}$ Flow Cytometry Unit, Life Sciences Core Facilities, Weizmann Institute of Science, Rehovot, \\ Israel
}

*Correspondence to: irit.sagi@ weizmann.ac.il, Department of Biological Regulation, Weizmann Institute of Science, Rehovot, IL-76100, Israel. Tel: +972-8-9342130

\footnotetext{
${ }^{\dagger}$ Present address: Wyss Institute for Biologically Inspired Engineering, Harvard University, Boston, MA, USA
} 


\begin{abstract}
Identification of early processes leading to complex tissue pathologies, such as inflammatory bowel diseases, poses a major scientific and clinical challenge that is imperative for improved diagnosis and treatment. Most studies of inflammation onset focus on cellular processes and signaling molecules, while overlooking the environment in which they take place, the continuously remodeled extracellular matrix. In this study, we used colitis models for investigating extracellular-matrix dynamics during disease onset, while treating the matrix as a complete and defined entity. Through the analysis of matrix structure, stiffness and composition, we unexpectedly revealed that even prior to the first clinical symptoms, the colon displays its own unique extracellular-matrix signature and found specific markers of clinical potential, which were also validated in human subjects. We also show that the emergence of this pre-symptomatic matrix is mediated by subclinical infiltration of immune cells bearing remodeling enzymes. Remarkably, whether the inflammation is chronic or acute, its matrix signature converges at presymptomatic states. We suggest that the existence of a pre-symptomatic extracellular-matrix is general and relevant to a wide range of diseases.
\end{abstract}

\title{
Keywords
}

Extracellular matrix, proteomics, electron microscopy, proteolysis, inflammation, colitis 


\section{Introduction}

The majority of research efforts on complex diseases are invested in studying cellular processes and cellular signaling molecules, e.g., cytokines, chemokines and growth factors, which often represent only a subset of factors responsible for tissue pathology. All of these processes and molecular signals are embedded within the extracellular matrix (ECM), which comprises a large portion of the tissue. Due to the intimate cell-matrix relationship, aberrations in the ECM hold the potential to induce pathological cellular behavior[1-4]. Recent studies suggest that dysregulated production of ECM modulators plays key roles in inflammation[5,6]. Along these lines, we hypothesized that the ECM can serve as an early reporter for multifactorial inflammatory diseases resulting in tissue damage. We thus set to investigate our hypothesis in inflammatory disease models in which tissue damage and ECM remodeling are known to occur[7].

Inflammatory bowel diseases (IBD) is an umbrella term for idiopathic diseases in the digestive tract, namely Crohn's disease (CD) and Ulcerative Colitis (UC). Early clinical intervention is key for preventing the cascading of chronic inflammation into irreparable tissue damage. However, this is challenging since, similarly to many idiopathic complex disorders, diagnosis is determined according to symptoms that appear well after molecular pathological processes are already taking place.

A number of studies over the past two decades have found ECM remodeling enzymes to be upregulated in human IBD. In a complimentary manner, the key role of protein and glycosaminoglycan composition of the ECM and its remodeling in the onset, progression and severity of IBD has been implicated in various rodent models[8-17]. We have previously provided preliminary evidence for the structural deformation taking place in the colonic ECM of 
IBD patients[7]. Accordingly, we set out to explore early involvement of ECM remodeling in the disease, before any gross signs of inflammation are detected.

In this study, we monitored ECM dynamics during intestinal inflammation by utilizing two common murine models for IBD - the acute dextran sodium sulfate (DSS)-induced colitis model[18], and the chronic piroxicam-accelerated colitis (PAC) model in interleukin(IL)-10 $0^{-/-}$ mice[19], and then validating them using available human specimens from healthy individuals and IBD patients. Through the analysis of matrix structure, stiffness and composition, we unexpectedly revealed a pre-symptomatic state with its own unique ECM signature and found markers of clinical potential. In addition, we show that this signature emerges, at least in part, by the increased activity of remodeling enzymes, especially basement-membrane degrading gelatinases, originating from infiltrating immune cells and the epithelium. Our integrated analysis highlights the ECM as a central component of early inflammatory processes and its potential as a prognostic biomarker.

\section{Results}

\section{Characterization of murine colitis models for mapping ECM properties and dynamics}

To probe the properties of native ECM during disease-associated transitions we adopted established murine models for IBD. Noteworthy, the study of IBD is almost exclusively immunological and cell-centric, with only limited study of the ECM and lack of information regarding its material properties, since it is usually relegated to background or contextual consideration. We have challenged this bystander role for the ECM[7].

To begin, we use a common murine model for IBD - the DSS-induced colitis model[18], which, with the appropriate calibration, results in a well-defined time-frame of acute colitis development in wild-type (WT) mice (Fig. 1A, see Experimental procedures for details). Disease 
progression was monitored by body weight measurement, endoscopic evaluation and histological analysis of the colon, in accordance with commonly used clinical diagnostic methods[20] at different time points - day 0 (healthy WT), day 4 and day 10 (Fig. 1 and Fig. S1, see Experimental procedures section for details on endoscopic scoring). Colon inflammation under these conditions peaks on day 10, as detected by endoscopy and histology (Fig. S1 and Fig. 1BD). Importantly, while clinical symptoms begin to appear after day 5, on day 4 most animals (over 90\%) are defined as "healthy" (Fig. 1C), since they do not lose weight (Fig. 1E), appear healthy by endoscopic evaluation, and do not display inflammation that is consistently detectable via histopathological analysis (Fig. 1B and 1D). Therefore, we regard day 4 as the "presymptomatic" state for analysis of ECM dynamics before the emergence of clinical symptoms.

Since our goal was to use a range of ECM states along the health-disease axis in intestinal inflammation, we also examined C57BL/6 IL-10-/- mice ("IL-10") that spontaneously develop chronic colon inflammation[21]. In these mice, in contrast to the DSS model, inflammation is not chemically induced, but rather is thought to result from immune dysregulation[22], and phenotypically, they more closely resemble the clinical manifestation of chronic UC and CD patients and mimic a monogenic form of IBD[19,23] (Fig. 1A, see Experimental procedures for details). IL-10 mice develop IBD-like symptoms, which can be detected and scored using colonoscopy, similarly to the well-established DSS-induced model (Fig. S1). Histopathology in this model shows prominent immune cell infiltration into the mucosa and has a different scoring system than DSS (see Experimental procedures for details) (Fig. 1B). Importantly, healthy naïve IL-10 mice do not display histopathological parameters of inflammation, while ill mice have a high histopathological score of 5.5 out of 8 on average (Fig. 1D). The penetrance of disease in this model is different than in the DSS-induced colitis, as just over $70 \%$ of the mice develop 
colitis at varying degrees, with a $20 \%$ mortality rate (Fig. 1C). For comparing acute (day 10 of DSS) and chronic inflammation, we selected a stable chronic-inflammation time point for further investigation (day 28), when mice appear to be at, or surpass, their initial weight before treatment (Fig. 1E). For comparison to the pre-symptomatic day 4 time point in the DSS model, we chose to use naïve eight to twelve-week-old IL-10 mice that were deemed "healthy" by endoscopic evaluation. Taken together, pre-symptomatic states and clear disease states were defined in two animal models: one for transient acute colitis (DSS) and the other for persistent chronic colitis (IL-10).

\section{ECM morphological changes precede inflammatory symptoms}

Having established pre-disease and disease state conditions, we sought to analyze the dynamics of ECM morphology by direct visualization. To do this, we first utilized secondharmonic generation (SHG) microscopy, which allows visualization of fibrillar collagen, without labeling, in native tissues. More detailed and high-resolution inspection of the spatial organization of the ECM was achieved using scanning electron microscopy (SEM) on decellularized colon tissue from the same mice.

Integration of SHG and EM analyses goes beyond the histological level, providing a detailed architectural depiction of the ECM in each tissue state namely, WT, pre-symptomatic, acute and chronic inflammation. Remarkably, marked structural and morphological changes in ECM could be observed already before any clinical or histological evidence of inflammation (Fig. 1). Below we describe each state and its unique architectural ECM signature.

\section{$\underline{\text { Healthy WT colonic ECM architecture }}$}

SHG microscopy revealed that in the colon of the healthy WT mouse, the collagen (Fig. 2A) uniformly circumscribes the colonic crypts with little to no signal of fibrillar collagen between crypt borders (borders are pointed out by arrows in Fig. S2A). The tissue architecture in 
this state is well-organized, with a homogeneous crypt diameter (mean $=53.7 \mu \mathrm{m}$, average $\mathrm{STD}=10.8 \mu \mathrm{m})$ and distance between crypts (mean $=12.8 \mu \mathrm{m}$, average $\mathrm{STD}=6.1 \mu \mathrm{m})($ Fig. $2 \mathrm{C})$. High-resolution SEM reveals that healthy crypts are uniformly covered by a dense mesh layer, which is most likely the basement membrane, according to its morphology[24-26] (Fig. 2B).

\section{$\underline{\text { Acute colitis ECM architecture }}$}

Both imaging modalities reveal that acute inflammation (day 10 of DSS) has a profound effect on ECM structure. This state presents with destruction of tissue architecture (Fig. 2A), altered and heterogeneous crypt size $($ mean $=78.7 \mu \mathrm{m}$, average $\mathrm{STD}=19.1 \mu \mathrm{m})$ and distance between adjacent crypts (mean=38.1 $\mu \mathrm{m}$, average $\mathrm{STD}=19.4 \mu \mathrm{m}$ ) (Fig. 2C), and disrupted ECM morphology (Fig. 2B and Fig, S2B), as well as regions containing the high-intensity fibrillar structures highlighted in Fig. S2A. These findings indicate that acute inflammation is characterized by regional ECM degradation and build up.

\section{Chronic colitis ECM architecture}

Similarly, SHG imaging of chronic inflammation in IL-10 mice reveals deterioration of collagen structure supporting the crypt wall, with crypt boundaries defined by a lower intensity signal than its corresponding healthy state (Fig. 2A), and the presence of disoriented fibrillar structure between crypts (Fig. S2A). The distance between adjacent crypts, as well as their diameters, increases and collagen ultrastructure is heterogeneous compared to the healthy IL-10 mice, from a mean diameter of $60 \mu \mathrm{m}$ to $85.3 \mu \mathrm{m}$ and from a mean crypt distance of $20.1 \mu \mathrm{m}$ to 37.8 $\mu \mathrm{m}$ (Fig. 2C and Fig. S2C-D).

Further inspection of the ECM structure using SEM reinforced this finding: naïve IL-10 crypt wall ECM appears fibrous, whereas in chronically ill IL-10 mice, the crypt wall ECM appears 
disintegrated and perforated, with fibrillary structures of different morphology (Fig. 2B and Fig. S2B).

\section{$\underline{\text { Pre-symptomatic colitis ECM architecture }}$}

Remarkably, at the pre-symptomatic state of the DSS model (day 4), thickening or deterioration of some crypt walls occurs, with changes in crypt diameter, along with the appearance of fibrillar structures in the spaces between crypts (Fig. 2A and 2C and pointed out by arrows in Fig. S2A), indicating remodeling events of the colonic ECM. Even more prominent ECM remodeling can be seen at this stage, using high-resolution SEM, as exposed fibrillar collagen on the inner crypt walls (Fig. 2B), due to destruction of the basement membrane mesh[25,26] coating the interstitial ECM (Fig. S2B).

Most surprising was the comparison of the healthy IL-10 mice to their WT counterpart and to day 4 of the DSS model. We observe a strong resemblance in ECM structure at highresolution between the colon of IL-10 mice and that of the pre-symptomatic, day 4 of DSS, with the characteristic exposure of fibrils on the crypt wall and disrupted basement membrane, as pointed out by arrows in Fig. 2B, and shown in more detail in Fig. S2B. To test whether this observation is phenomenologically robust, we quantified the frequency of fibril exposure in the two pre-symptomatic states (day 4 of DSS and healthy IL-10), as a percentage of crypts that have exposed walls, by comparing to the frequency in healthy WT mice. We found that this structural change occurs in both models at a statistically significant rate, of $\sim 90 \%$ for day 4 and $\sim 75 \%$ for healthy IL-10 (Fig. 2D) compared to healthy WT ( 6\%). Thus, IL-10 mice, which are prone to developing chronic inflammation, develop an injured basement membrane as a quasi-stable presymptomatic state, compared to the dense mesh covering the WT murine colon (Fig. 2B and Fig. S2B). 
Taken together, the structural similarity of the ECM between the two murine presymptomatic states, leads us to hypothesize that exposed fibrillar collagen due to the deterioration of the basement membrane, is one of the features of what we term the "presymptomatic ECM".

\section{Disease onset is associated with a gradual loss of matrix rigidity}

Having defined the structural and morphological differences of the matrix in presymptomatic states, we applied a second dimension of our analysis, which interrogates the changes in the mechanical properties of the matrix. To do this, we used mesoscale atomic-force microscopy (AFM) on decellularized ECM scaffolds derived from colons of mice from both experimental models, using pre-symptomatic (day 4 of DSS and healthy IL-10) and pathological (day 10 of DSS and chronically ill IL-10) states, in addition to healthy WT.

We used micron-sized probes to apply pressure to a $5 \mathrm{mmX} 5 \mathrm{~mm}$ (hydrated size) ECM scaffold. This permitted acquisition of the scaffolds' mesoscopically averaged, statistically robust Young's Modulus (YM) values[27,28], which describe the collective contributions of nanoscale molecular components organized in micrometer-sized $(70 \mu \mathrm{mX} 70 \mu \mathrm{m})$ domains in the ECM (see Experimental procedures for more details). Distributions of the measured YM values of colonic ECM samples are broad (Fig. S3), demonstrating the diversity of elastic properties within the same sample, and suggesting variability within the complex structure and composition of the ECM of each individual. Each mode in the distribution (Fig. S3 and Table S2) represents the elastic properties of a region of the ECM with lateral dimensions (and thickness) of several microns, which we term "mechanical domains". The median values and average median values of the YM of these mechanical domains are reported in Fig. 2E. 
The rigidity of the ECM in healthy mice, both WT mice and IL-10, is similar and relatively high, with median YM values of about $11 \mathrm{kPa}$. As inflammation is taking place, the colonic ECMs are generally softer than those of healthy colons, as indicated by the median YM (Fig. 2E and Table S1). Importantly, the structurally modified ECM in the pre-symptomatic state (day 4) of the DSS model has mechanical domains with an intermediate rigidity $(\mathrm{YM}=9 \mathrm{kPa})$, with values between those of healthy $(\mathrm{YM}=10.5 \mathrm{kPa})$ and acutely inflamed $(\mathrm{YM}=6 \mathrm{kPa})$ states of DSS. The ECM of the chronically ill IL-10 mice (YM=3kPa) is much softer than that of the healthy IL-10 mice (YM=11kPa) (Fig. 2E and Table S1). Remarkably, chronically ill IL-10 mice possess a YM value that is approximately two-fold lower than that of the acutely inflamed colon (Fig. 2E and Table S1).

Altogether, our two-pronged analysis highlights a gradual softening of the ECM over the course of acute colitis development, peaking along with acute symptoms, which are accompanied by massive structural ECM damage.

\section{Matrisome protein compositional dynamics reflects the state of the colon}

To understand the molecular outcome of the remodeling events that are evidently taking place in the ECMs of the two distinct pre-symptomatic states, we analyzed their protein composition - the matrisome[29]. We used mass-spectrometry (MS)-based analysis of the tissue proteome, followed by statistical analysis and computational modeling. Eight to ten colon samples from individual mice were analyzed from each of the five states: healthy WT, the two pre-symptomatic states (i.e., day 4 DSS, healthy IL-10), and two types of intestinal inflammation (acutely ill day 10 of DSS, chronically ill IL-10). Out of 2045 total proteins identified in all states, 110 ECM and ECM-related proteins, comprising 5\% of all identified proteins, were 
retrieved. Annotation as an ECM protein was made according to the Matrisome, an ECM protein cohort defined by Naba et al [29].

We identified the ECM proteins that significantly change in abundance among states (see Experimental procedures for details). A scheme summarizing our results is shown in Fig. 3A, and in more detail in Table S3. We found that the ECM composition of the healthy WT state is very different from that of both states of illness. We also detected a statistically significant proteomic compositional shift in both pre-symptomatic states compared to the healthy WT. A large portion of this protein abundance shift between WT and pre-symptomatic states (i.e., increase in Collagen alpha-2(I) chain, Fibrillin-1 and Transglutaminase-3; decrease in Mucin-2 and Lectin mannose-binding-1) is shared between the two pre-symptomatic states (Fig. 3B). Remarkably, these two states exhibit the highest compositional similarity of any pair, with no differentially abundant ECM proteins at all (Fig. 3A). Moreover, the matrisomic shift from each pre-symptomatic state to its corresponding ill state also shows a shared signature, which includes the reduction of several structural components (e.g., collagens, Laminin and Fibulin-5) (Fig. 3C), corresponding to the structural damage observed in ill states (Fig. 2). The two ill states, though clinically and functionally distinct, are also quite similar in terms of differentially abundant proteins, as summarized in Fig. 3A.

To bridge the gap between omics data and tissue morphology and function, we further analyzed the data holistically, using unsupervised learning tools. These tools provide an unbiased analysis of the data, based on the variance of the input data, and not on the assigned identity, or clinical state of each sample. We identified three clusters of proteins with similar abundance patterns (Fig. 3D). We then used principle component analysis (PCA) on the means of each cluster in order to rank the different states according to their protein composition. Our analysis 
led to the formation of PC1, which is dominated (75\%) by protease inhibitors (e.g., serpins) and coagulation factors (e.g., subunits of the fibrinogen complex), while the rest is composed from ECM structural proteins (e.g., collagens, proteoglycans and glycoproteins) (Table S4). PC1 reflects the tissue damage and ECM destruction characteristic of disease states. Strikingly, PC1 ranked the samples from the healthy WT to pre-symptomatic states (first healthy IL-10 and then day 4 DSS), followed by chronically ill IL-10 and finally, the acutely ill, DSS day 10 state. Thus differentiation of samples mostly according to the abundance of proteins in cluster 1 is sufficient to correctly distinguish between the five different states (Fig. 3E). The two states at the edges of this ECM ranking, or hierarchy, represent the two extremes of their clinical states. We note that healthy IL-10 (as a chronic pre-symptomatic state) is closer to the "healthy edge" than the transient pre-symptomatic state of day 4 of DSS. This observation is consistent with the ECM rigidity analysis, described above.

In summary, the compositional analyses of all experimental samples provides a molecular signature for the "pre-symptomatic ECM" in both acute and chronically ill models, which is compositionally distinct from healthy WT or ill states, but is similar between inflammation models. Furthermore, the compositional healthy-ill ranking arising from our unbiased computational analysis corresponds to the degree of structural damage that we observed via imaging while independently highlighting the existence of the pre-symptomatic state.

\section{Human IBD recapitulates murine matrisomic dynamics and validates biomarkers}

In order to assess the clinical relevance of the identified biomarkers, we first tested whether the murine ECM proteomic analysis could be confirmed using an independent human dataset. One existing proteomic dataset comparing healthy donor biopsies with those of UC patients, deposited in the ProteomeXchange, was used for this experiment[30]. We examined the 
relative abundance levels of the proteins that were significantly differentially expressed between the healthy mice (both WT and IL-10) and their corresponding fully diseased sample types (Fig. 4A). A significant proportion of these proteins exhibit the same trends in relative abundance in the human dataset: proteins that are more abundant in ill mice than healthy mice are also more abundant in UC patients than in healthy donors, and vice versa (Fig. 4B). 
In order to search for distinguishing features that can serve as potential biomarkers in our ECM proteomics data, we have utilized a machine-learning classification algorithm for analyzing our dataset. Identification of such distinguishing features would depend upon correct automated classification of samples into either "healthy WT", "pre-symptomatic" or "ill" classes. The resulting model is illustrated in Fig. 4C (see legend for more details). The algorithm "chose" two proteins, Collagen XVIII/Endostatin (murine gene name: Col18a1) and Fibrillin-1 (murine gene name: Fbn1), as the nodes of the tree. Collagen XVIII is a ubiquitous non-fibrillar collagen, and a crucial, structurally complex basement membrane proteoglycan[31-33]. The glycoprotein Fibrillin-1, is part of the microfibril-forming Fibrillin family that is important for connective tissue integrity and function[34,35]. Plotting all samples according to the abundances of these two proteins resulted in a clear separation between the healthy, pre-symptomatic and ill states (Fig. 4D). Of note, the pre-symptomatic state is defined by low levels of Col18a1 and high levels of Fbn1. Real-time qPCR analysis corroborated our findings regarding Col18 while no significant change in Fbn1 expression could be observed, suggesting that upregulation of Col18a1 occurs at the transcriptional level, while Fbn1 is most likely regulated at the protein level (Fig. 4E). Remarkably, these observations of protein and transcript abundance exhibit similar patterns of relative abundance in clinically isolated human biopsies, as described below.

Next, we examined expression of these same putative biomarkers in a new set of human biopsies. For this, we compared mRNA expression levels of the human homologs, COL18A1 and FBN1, between healthy donor biopsies and tissues samples from IBD patients that were endoscopically and histologically identified as inflamed (sample list in Table S6). We found that both COL18A1 and FBN1 are significantly upregulated, in concordance with the decision tree applied to the proteomic data from our murine colitis samples (Fig. 4F). To corroborate this 
finding on the protein level and tissue localization, we also stained for Fibrillin-1 in inflamed rectal biopsies of pediatric UC patients with distal colitis (sample list in Table S7). We have found that a subset $(\sim 50 \%)$ of these patients display a strong epithelial staining of Fibrillin-1 ("FBN1 positive"), while the rest look like the control with little to no staining (Fig. 4G-H).

Taken together, analysis of published human proteomic datasets, qPCR and immunostaining of clinical biopsies, validate the clinical potential of the biomarkers Collagen XVIII and Fibrillin-1, which we identified using a classifier algorithm.

Pre-symptomatic ECM changes coincide with epithelial gelatinase activity, Laminin degradation and immune cells bearing remodeling enzymes

Having observed that the most prominent structural ECM alteration, along with ECM softening, occurring at pre-symptomatic states involves injury, and hence - degradation of the basement membrane underlying the epithelial cells of the colonic crypts and covering collagen fibrils, we set to examine the levels and cellular sources of mostly ECM proteases and gelatinolytic activity in these tissues. First, we tested mRNA expression in whole tissue specimens of both gelatinases-A and -B (MMP-2 and MMP-9), which primarily degrade basement membranes[36,37]. We also tested other MMPs, LOX (a collagen crosslinking enzyme) and the tissue inhibitor of MMPs (TIMP)-1. We found that both MMP-2 and -9 were predominantly upregulated both at day 4 of the DSS model and in healthy IL-10 mice compared to healthy WT, and that the expression of TIMP-1, which inhibits MMP-2 and -9, was unchanged (Fig. 5A). Since the final regulatory step of MMPs that determines their net contribution to ECM remodeling[38] occurs outside of the cell, with their activation, we used the activity-based assay, in situ zymography[39] to localize active gelatinases in the tissue. Our analysis reveals that elevated ( 1.5-2 fold) gelatinolytic activity is detected in the crypts of the 
pre-symptomatic tissues, compared to healthy WT (Fig. 5B-C), and appears to localize to the epithelium. To further examine the effect of gelatinase activity on one of its substrates in the basement membrane, we stained colon cross sections for Laminin, comparing healthy WT to the two pre-symptomatic states. The Laminin structure in the mucosa of healthy WT, has a compact appearance, with distinct high intensity linings at crypt borders. In contrast, in pre-symptomatic states, especially in day 4 samples, Laminin has a more diffuse appearance, indicating structural damage (Fig. 5D). We quantified this difference by measuring the intensity of high frequencies of the Fourier Transform of the images, which correspond to the sharpness of the image (i.e., where signal intensity changes more rapidly), and found that healthy WT laminin staining is indeed sharper than it is in pre-symptomatic states, most significantly day 4 of DSS (Fig. 5E). Finally, we also performed a degradomic analysis to see what proteins undergo degradation and found that subunits of Laminin (significantly Lamb2, and a trend in Lamc1), along with Collagen VI and Vitronectin, are more degraded in day 4 of DSS than in healthy WT (Fig. 5F).

In order to investigate the cellular sources of ECM remodeling enzymes at the presymptomatic states, we used mass-cytometry analysis that allows us to sort cells according to a large number of markers. This yields a comprehensive picture of the relative frequencies of different cell populations that express proteins of interest. The results reveal that despite showing no signs of inflammation by standard clinical tools[20], and not having a significant change in the total proportion of immune cells in the tissue (Fig. S5A), mice on day 4 display a statistically significant elevation in the percentage of neutrophils ( $0.5 \%$ of live cells) and monocytes $(0.3 \%$ of live cells) in the colon compared to healthy WT $(0.1 \%$ and $0.07 \%$ of live cells, respectively (Fig. 6A and Fig. S5B). This finding demonstrates the earliest step of immune cell infiltration. In addition, though also not displaying clinical and histopathological signs of inflammation, healthy 
IL-10 mice have a larger proportion of immune cells, consisting mainly of populations from the adaptive immune response and those related to antigen presentation ( $\mathrm{T}$ helper cells, B cells and dendritic cells), compared to healthy WT mice. These different immune cell populations in the two different pre-symptomatic states carry a variety of ECM remodeling enzymes, especially the basement-membrane degrading gelatinases MMP-2 and MMP-9 (Fig. 6B-G). This indicates the contribution of subclinical immune cell infiltration to the formation of the pre-symptomatic ECM, and the observed proteolytic activity in Fig. 5B. These findings suggest that MMP-9, an established marker of active IBD[16,40], displays elevated activity at pre-clinical states as well, most pronouncedly in healthy IL-10 mice, where the total number of MMP-9 positive cells is significantly higher than in its WT counterpart (Fig. S5C). The data also reveals that MMP-2expressing epithelial cells account for $\sim 20-30 \%$ of all living cells, and though this proportion remains constant also at pre-symptomatic states (Fig. S5D), their contribution of MMP-2 may be augmented via changes in expression levels per cell or activation levels (rather than the number of cells expressing it). In addition, we found that at day 4 of the DSS model, innate immune cells (i.e., neutrophils, monocytes, macrophages and dendritic cells) carry LOX, a collagen crosslinking enzyme, which may explain the thickening of the crypt walls at this presymptomatic state.

Together with the multifaceted analysis of the previous sections, we show that elevated ECM enzymatic degradation, especially of the basement membrane, from various cellular sources takes part in the structural ECM alterations that we observe at pre-symptomatic states.

\section{Discussion}

The "pre-symptomatic ECM", which we have identified and characterized, on multiple levels of analysis herein, is a reproducible signature that is virtually identical in acute and 
chronic colitis models, and crucially, is distinct from that of the healthy WT tissue. Our analysis exposed several aspects and processes of remodeling, which potentially contribute to setting the stage for inflammation and understanding the nature of the challenge it imposes on the tissue.

Specifically, we identify the existence of a silent inflammatory tissue state by combining structural, mechanical and molecular system-level ECM analyses. We reveal that significant ECM remodeling precedes the appearance of detectible clinical symptoms, which is characterized by structural damage, especially to Laminin comprising the basement membrane, and changes in stiffness and proteomic composition. This remodeling is accomplished both by elevated production and activity of remodeling enzymes in the epithelium, along with subclinical immune-cell infiltration, which cannot be detected by conventional histopathological analysis. Interestingly, though the transiency of this state in each model is different and involves different immune cell populations contributing remodeling enzymes, the effect on the ECM is similar. In addition, a recent report indicates that this pre-symptomatic ECM, in the specific case of the DSS colitis model, can be achieved via direct basement membrane damage inflicted by DSS[41], supporting our observation that basement membrane damage is part of the injury process in this model. Our unsupervised analysis of ECM compositions also showed that by narrowing our proteomic results into principal components (Fig. 3E) we can distinguish the pre-symptomatic state from all other states in the colitis model, enhancing our view that this is indeed a separate entity. Finally, our preliminary human IBD characterization recapitulates our observation of murine matrisomic dynamics and validates the found biomarkers.

Another discrete feature of the pre-symptomatic state highlighted by our characterization arises from the supervised learning analysis on the matrisome, which suggests a convergence of the five states examined into 1) healthy WT, 2) pre-symptomatic, and 3) ill. We identified 
Collagen XVIII/Endostatin and Fibrillin-1 as features distinguishing these states - indicating either ill or pre-symptomatic states, respectively. Neither of these proteins has been extensively studied in the context of IBD previously. Recently, a single report showed that upregulation of the FBN1 gene expression at first diagnosis of pediatric IBD predicts the fibrostenotic complication of the disease[42]. This suggests the subset of patients with FBN1-positive epithelial staining at first diagnosis in our study as candidates for long-term clinical follow-up, to determine whether this molecular ECM feature may have a similar prognostic impact. Thus, machine learning as part of our compositional analysis has already demonstrated its potential for identifying relevant clinical markers and disease prognostic indicators.

Though IBD pathophysiology involves major tissue-damaging processes, which ultimately lead to the complications of the diseases[43-46], little is known to date regarding tissue remodeling events involving ECM before clinical presentation in these diseases. Scheme 1 summarizes our observed pre-symptomatic signatures, and illustrates how they may contribute to the promotion of inflammation.

Importantly, along with the convergence of pre-symptomatic states in two different colitis models, our findings are in line with current knowledge, stating that neutrophils bearing MMP-9 are often the first immune cell type to initiate the acute inflammatory process[47,48], and that the basement membrane is a key player in the barrier function of all mucosal tissues[49,50]. These strongly suggests that a similar pre-symptomatic ECM would exist in other inflammatory diseases, such as in the airway or other parts of the gastrointestinal tract. In addition, though the colitis in the IL-10 $10^{-/-}$mouse model is well characterized, including dysbiosis[51] and the immune cell populations involved[22],little is known about the onset of symptoms. One study showed that altered mucin production before symptom presentation may be involved in IL-10 ${ }^{-/}$colitis 
onset[52], which is consistent with our proteomics results showing that Muc-2 levels are lower in healthy IL-10 mice compared to WT. Here we also show that though immune cell infiltration cannot be observed in healthy IL-10 mice via histology, the colons of these mice have higher numbers of different immune cell populations, both adaptive and innate that carry ECM remodeling enzymes, compared to their WT counterparts. Therefore, we propose that the disruption to ECM integrity by a small number of immune cells, and the secretion of gelatinases by the epithelium, allows access to additional immune cell infiltration and bacterial antigens, which creates a tissue that can readily be "pushed" towards full-blown inflammation. In summary, our integrative approach permits a well-rounded characterization, encompassing multiple levels of detail, of the ECM - a complex and substantial material that comprises tissues. We conclude that by manifesting the pathophysiological state of a tissue, the ECM, in all of its complexity and properties, reveals silent disease-prone states that occur before clinical symptoms. The lessons from the observations we made in intestinal inflammation can be applied to other scenarios of inflammation due to the similarities of its onset in other mucosal tissues. We anticipate that this work will fuel mechanistic studies on the means by which early morphological and biomechanical ECM remodeling events leave the tissue vulnerable or prone to other pathologies such as cancer, fibrosis, and inflammation in other organs. Identification of such additional silent pathological states by utilizing the ECM as a reporter will contribute to early diagnosis, managing relapse and intervention in tissue-damaging diseases.

\section{Experimental procedures}

\section{$\underline{\text { Animals }}$}

Seven-week old C57BL/6 male mice were purchased from Envigo and were allowed to adapt for one week before experimental procedure. IL-10 ${ }^{-/-}$C57BL/6 mice from Jackson 
Laboratories were inbred at the Weizmann Institute of Science, and experiments were performed on six to eight-week old male mice. Healthy naïve IL-10 ${ }^{-/}$were eight to twelve weeks old at time of harvest. All experiments and procedures were approved by the Weizmann Institute of Science animal care and use committees, protocol numbers 02230413-2, 06481012-1.

\section{$\underline{\text { Human samples }}$}

Biopsies were collected from the colonic mucosa of healthy donors and of IBD patients after providing informed consent in accordance with the ethical standards on Human Experimentation and the Declaration of Helsinki (protocol \# 0725-14-TLV and \# 3312-16SMC). Samples were classified as being inflamed or normal by endoscopy and histology. Surgically resected inflamed colon samples from IBD patients were obtained from the Israel National Biobank (MIDGAM).

Samples were taken from both male and female subjects. The samples in each group for the RNA analysis are listed in Table S6. Samples used in RNA analysis were snap-frozen in liquid nitrogen until RNA extraction. Biopsies used for immunohistochemical stains were fixed in $4 \%$ paraformaldehyde and the sample list is in Table S7.

Intestinal inflammation induction and evaluation

Acute colonic inflammation was induced by administration of $1.25 \%$ DSS (MP Biomedicals LLC) in drinking water of C57BL/6 mice for seven days[18]. Chronic colonic inflammation was accelerated and synchronized by peroral administration of 200ppm piroxicam (Sigma-Aldrich ltd.) to IL-10 ${ }^{-/-}$C57BL/6 mice via supplementation in normal murine chow[19]. Mice were weighed 3 times a week over the course of the experiment. Colitis progression was evaluated over the course of the experiment using the Karl Stortz Coloview mini endoscope system and colonic inflammation was scored (0-15) as previously described[53]. Inflammation scores were 
categorized according to the following: healthy (0-4); mildly inflamed (5-7); inflamed (8-11); severely inflamed (12-15). Another form of inflammation evaluation was histological analysis by H\&E staining of formalin-fixed paraffin-embedded tissues sections. The degree of histological damage and inflammation was graded in a blinded fashion by an expert pathologist. The following parameters were scored in the DSS-induced colitis model: amount of inflammation (0, none; 1 , mild; 2 , moderate; 3 , severe; 4 , accumulation of inflammatory cells in the gut lumen); percentage of colon area involved ( 0 , none; $1,<=10 \% ; 2,10 \%-30 \% ; 3,30 \%-50 \% ; 4,50 \%-90 \%$; $5,>90 \%)$; depth of inflammation and layers involved ( 0 , none; 1 , mucosa only; 2 , mucosa and submucosa; 3 , limited transmural involvement; 4 , transmural $)$, ulceration $(0,<30 \%$; $+1,>30 \%$; 1, regeneration). The overall histological score was the sum of the three parameters (maximum score of 14). Histopathological scoring for the IL-10 model was performed in a similar fashion, but only considering the amount of inflammation and percentage of colon area involved (maximum score of 9). IL-10 mice lose weight only in early phases of colitis development, and the weight is regained as chronic colitis is established (Fig. 1E).

Mass cytometry and cell sorting

Cell isolation of colon samples was performed as previously described[54]. In brief, colons were harvested on the day of the experiment from healthy WT mice, healthy IL-10 mice, and mice of day 4 of the DSS model, were carefully separated from the surrounding fat and lymphatic vessels and cleaned from feces with cold PBS. Then, $2 \mathrm{~cm}$ colon pieces were minced and immersed into RPMI-1640 medium (GIBCO, 21875034) containing 10\% FBS, 2 mM HEPES, 0.05mg DNase (Roche, 04716728001), and $0.5 \mathrm{mg} / \mathrm{mL}$ of collagenase VIII (SigmaAldrich, C2139) and placed for 40 minutes at $250 \mathrm{rpm}$ shaking at $37^{\circ} \mathrm{C}$ and mashed through a $70 \mu \mathrm{m}$ cell strainer. 
Following, cells were stained according to a previously published protocol[55]. Individual mice cell suspensions were stained with Cell-ID Cisplatin $0.125 \mu \mathrm{M}$ for viability and fixed using Maxpar® Fix I Buffer. Samples were then permeabilized using Maxpar® Barcode Perm Buffer and then barcoded using The Cell-ID ${ }^{\mathrm{TM}}$ 20-Plex Pd Barcoding Kit, allowing us to join samples for antigen staining. Antibodies used for staining are listed in Table S8. Before analyzing, the cell suspension was incubated with Cell-ID Interculator Iridium for 20 minutes.

Cells were analyzed with a cyTOF2® mass cytometer (Fluidigm). Results were normalized and debarcoded using Fluidigm cyTOF software[56]. CyTOF results gating and further analysis was done with FlowJo software (FlowJo, LLC) and cell populations were defined according to the markers listed in Table S9. tSNE analysis was done using the viSNE application in the Cytobank web platform. tSNE (t-Distributed Stochastic Neighbor Embedding) is a machinelearning algorithm used to cluster multivariate data into a 2D representation.

$\underline{\text { Decellularization of colonic tissue }}$

Decellularization was performed as previously described[57]. Samples were frozen in $\mathrm{ddH}_{2} \mathrm{O}$ and subjected to six freeze-thaw cycles with replacement of $\mathrm{ddH}_{2} \mathrm{O}$ each time. Subsequently, samples were immersed in $25 \mathrm{mM} \mathrm{NH} 4 \mathrm{OH}$ (Merck) for 20 minutes. Finally, isolated ECM scaffolds were washed six times in $\mathrm{ddH}_{2} \mathrm{O}$ and stored at $4^{\circ} \mathrm{C}$ until use.

\section{$\underline{\text { Second-harmonic generation (SHG) microscopy }}$}

Native snap-frozen murine colon samples were thawed in PBS and imaged using a twophoton microscope (2PM:Zeiss LSM 510 META NLO; equipped with a broadband Mai Tai-HPfemtosecond single box tunable Ti-sapphire oscillator, with automated broadband wavelength tuning 700-1,020 nm from Spectraphysics, for two-photon excitation). For second-harmonic imaging of collagen, a wavelength of $800-820 \mathrm{~nm}$ was used (detection at 390-450nm). 
Quantification of crypt diameter was done using ImageJ software (Research Service Branch, $\mathrm{NIH})$.

\section{$\underline{\text { Scanning electron microscope (SEM) }}$}

Decellularized mouse colon tissues were used and prepared as described in [58]. Then, samples were subjected to critical point dehydration (CPD) and attached to a carbon sticker. Finally, samples were coated with gold palladium, and imaged under SEM Ultra (Carl Zeiss international).

\section{$\underline{\text { In situ zymography }}$}

In situ zymography was conducted as previously described[39] on unfixed $10 \mu \mathrm{m}$ transverse mouse colon sections using DQ Gelatin ${ }^{\mathrm{TM}}$ (Invitrogen) from pig skin. Following, sections were fixed with $4 \%$ paraformaldehyde, and cell nuclei were stained with DRAQ5 (Thermo Scientific). Finally, mounting solution (Immu-Mount ${ }^{\mathrm{TM}}$ Thermo Scientific) was added and slides were covered with a coverslip. The slides were imaged under a Leica TCS SP8 microscope (both confocal and multiphoton). DQ gelatin was excited at 488nm and its emission was detected at $515 \mathrm{~nm}$.

\section{$\underline{\text { Testing elastic properties of ECM samples by Atomic Force Microscopy }}$}

The AFM analysis was carried out on rehydrated $5 \mathrm{mmX} 5 \mathrm{~mm}$ ECM samples derived from mouse colon tissues. Typically, three-four samples were analyzed for each inflammatory condition. The rehydration process was as follows: ECM samples were grossly dried and attached to glass coverslips (diameter $15 \mathrm{~mm}$ ) by means of a thin bi-adhesive tape. Samples were then attached to the bottom of Petri dishes (Greiner Bio-One) and left overnight in an evacuated desiccator in order to dry out, so to improve spreading and adhesion of ECM on tape. Prior to AFM measurements, the Petri dish hosting the ECM sample was filled with $\mathrm{ddH}_{2} \mathrm{O}$ and the ECM 
was allowed to rehydrate for 30 minutes at room temperature. Measurements were carried out at room temperature.

To accomplish the nanomechanical characterization of the ECM samples, a Bioscope Catalyst AFM (Bruker) was used to collect series of force vs distance curves[59,60], recording the local deformation of the sample induced by the AFM probe. According to a recently published protocol[28], we used monolithic borosilicate glass probes consisting in micrometersized spherical glass beads with radius $\mathrm{R}=9-10 \mu \mathrm{m}$ attached to silicon cantilevers with a force constant $\mathrm{k}=0.25-0.35 \mathrm{~N} / \mathrm{m}$. The probes were produced and characterized as previously described[27].

Each set of force curves (a force volume) consisted of a 16x16 array of curves acquired on a $70 \mu \mathrm{m} \times 70 \mu \mathrm{m}$ area. Ten force volumes were typically recorded on each ECM on macroscopically separated regions. All measurements were performed with the following parameters: 4096 points per curve, ramp length $L=10 \mu \mathrm{m}$, maximum applied force $F=60-70 \mathrm{nN}$, and ramp frequency $f=1.10 \mathrm{~Hz}$. Typically, indentations up to $2-3 \mu \mathrm{m}$ were obtained. Data processing of force volumes was carried out in Matlab according to the general protocol described previously[28]. The values of the Young's Modulus (YM) were extracted by fitting the Hertz model to the experimental data[59,60]. A first very soft indentation region $(0-40 \%$ of total indentation) was excluded, in order to separate the possible contribution of loosely-bound superficial layers from those of the bulk ECM. Artifacts derived from an ill-defined contact area between sample and probe, like boundaries of colonic crypts or more generally crypts with characteristic dimensions comparable to, or larger than the probe diameter, were identified and discarded. 
The distributions of YM values of the ECMs from mice turned out to be the envelope of several nearly lognormal modes, representing the major contributions to the overall ECM rigidity and originating from micro-scale domains that the AFM probe was able to resolve. Each mode in the distributions represents the elastic properties of a region of the ECM with lateral dimensions (and thickness) of several microns, which may define a structural and functional domain of the ECM. Multi-Gaussian fit in semilog10 scale allowed identification of the peak value E' and the geometric standard deviation $\sigma_{\mathrm{g}}^{10}$ of each lognormal mode; from these values the median value $E_{\text {med }}$ and the standard deviation of the median $\sigma_{\text {med }}$ were evaluated for all modes as $\mathrm{E}_{\text {med }}=10^{\mathrm{E}^{\prime}}$ and $\sigma_{\text {med }}=\frac{\sqrt{\frac{\pi}{2}} \mathrm{E}_{\mathrm{med}} \sigma_{\mathrm{g}}^{10}}{\sqrt{\mathrm{N}}}[61], \mathrm{N}$ being the number of force curves in each mode. The effective rigidity of each ECM sample was characterized by the weighted average of median values $\mathrm{E}=\sum_{\mathrm{i}} \mathrm{f}_{\mathrm{i}} \mathrm{E}_{\text {med,i }}$ using the fraction $\mathrm{f}_{\mathrm{i}}=\frac{\mathrm{N}_{\mathrm{i}}}{\mathrm{N}_{\text {tot }}}$ of force curves in the mode as the weight; the total error $\sigma_{\mathrm{E}}$ associated to $\mathrm{E}$ was calculated by summing in quadrature the propagated error of the medians $\sigma=\sqrt{\sum_{\mathrm{i}} \mathrm{f}_{\mathrm{i}}^{2} \sigma_{\text {med,i }}^{2}}$ and an effective instrumental relative error $\sigma_{\text {instrum }}=3 \%$ : $\sigma_{\mathrm{E}}=\sqrt{\sigma_{\text {instrum }}^{2} \mathrm{E}^{2}+\sigma^{2}}$. The average median values of the $\mathrm{YM}$ of the different states of inflammation have also been evaluated; the corresponding error has been calculated as the standard deviation of the mean summed in quadrature with the propagated $\sigma_{\mathrm{E}}$.

ECM proteomics by liquid chromatography-tandem mass spectrometry (LC-MS/MS) analysis

Tissue slices from colons of WT or IL-10 mice at different stages of the inflammation models were immersed in a solution containing $50 \%$ trifluoroethanol (TFE), $25 \mathrm{mM}$ ammonium bicarbonate (Sigma Aldrich) and $15 \mathrm{mM}$ dithiothreitol (DTT) and coarsely homogenized by 
repeated cycles of boiling, freezing and sonication. Subsequently, samples were shaken at $30^{\circ} \mathrm{C}$, $1400 \mathrm{rpm}$ for 30 minutes, followed by the addition of iodoacetamide (Sigma Aldrich) to a final concentration of $25 \mathrm{mM}$ and further shaking for 30 minutes at $30^{\circ} \mathrm{C}$ and $1400 \mathrm{rpm}$. TFE was then diluted to $25 \%$ with $50 \mathrm{mM}$ ABC, LysC (Wako Chemicals, 1:100 lysC:protein ratio) and sequencing-grade modified trypsin (Promega, 1:50 trypsin:protein ratio) was added and incubated overnight at room temperature. On the following morning, more trypsin was added (1:80 trypsin: protein ratio) for 4 hours. Peptide mixtures were purified on C18 stage tips. Eluted peptides were loaded onto a $50 \mathrm{~cm}$ long EASY-spray reverse phase column and analyzed on an EASY- nLC- 1000 HPLC system (Thermo Scientific) coupled to a Q-Exactive Plus MS (Thermo Scientific). Peptides were separated over 240 minutes with a gradient of 5-28 \% buffer B (80\% acetonitrile and $0.1 \%$ formic acid). One full MS scan was acquired at a resolution of 70,000 and each full scan was followed by the selection of 10 most intense ions (Data dependent Top 10 method) at a resolution of 17,500 for MS2 fragmentation.

Raw MS data was analyzed with MaxQuant software[62,63] (version 1.5.2.18) with the built-in Andromeda search engine[64] by searching against the mouse reference proteome (UniprotKB,Nov2014). Enzyme specificity was set to trypsin cleavage after lysine and arginine and up to two miscleavages were allowed. The minimum peptide length was set to seven amino acids. Acetylation of protein $\mathrm{N}$ termini, deamidation of asparagine and glutamine, and oxidation of methionine were set as variable modifications. Carbamidomethylation of cysteine was set as a fixed modification. Protein identifications were sorted using a target-decoy approach at a false discovery rate (FDR) of $1 \%$ at the peptide and protein levels. Relative, label-free quantification of proteins was performed using the MaxLFQ algorithm integrated into MaxQuant environment with minimum ratio count of two[65]. 


\section{$\underline{\text { Computational analysis of MS data }}$}

Classification was done using a J48 decision tree algorithm[66] with a stratified 10-fold cross-validation in the Weka software for machine learning (University of Waikato). As input for this classifier, we used the ECM proteomic data (i.e., 110 matrisome proteins) described above, comprising 8 healthy WT samples, 16 pre-symptomatic samples (day 4 of DSS and healthy IL10) and 20 ill samples (day 10 of DSS and ill IL-10), where each sample came from a different mouse. Clustering according to squared Euclidean distance and principal component analysis (PCA) were performed in Matematica (Wolfram Research).

$\underline{\text { Terminal amine isotopic labeling of substrates (TAILS) degradomic analysis }}$

For terminal amine isotope labeling of substrates (TAILS) experiments, $\mathrm{N}=4$ biological replicates (one $\mathrm{N}$ represents a colon sample from a single mouse as a biological replicate per time point) were snap frozen in liquid nitrogen. Prior to protein denaturation and labeling, proteins of samples were extracted by mechanical homogenization using Tissuelyser (QIAGEN) and sonication using Bioruptor Pico (Diagenode). This process was done on $10 \mathrm{mg}$ tissue in a buffer containing 4 M Guanidine hydrochloride (Sigma-Aldrich), 250 mM HEPES (SigmaAldrich (pH 7.8)) and protease inhibitor cocktail (cOomplete EDTA-free, Roche).

TAILS analysis, whereby protein N-termini were enriched, was performed according to the previously described protocol[67], except for using 16plex Tandem mass Tag ${ }^{\text {TM }}$ labeling (TMT, \#90110, Thermo Fisher) and adding the trypsin at a ratio of 1:20 (trypsin/protein ratio).

PreTAILS and TAILS peptide mixtures were resuspended in MS injection buffer (1\%TFA, 2\% Acetonitrile, containing iRT peptides (Biognosys)) loaded onto an PepMap100 C18 precolumn $(75 \mu \mathrm{m} \times 2 \mathrm{~cm}, 3 \mu \mathrm{m}, 100 \AA$, nanoViper, Thermo Scientific) and separated on a PepMap RSLC C18 analytical column $(75 \mu \mathrm{m} \times 50 \mathrm{~cm}, 2 \mu \mathrm{m}, 100 \AA$, nanoViper, Thermo 
Scientific) using an EASY-nLC 1200 liquid chromatography system (Thermo Scientific) coupled in line with a Q Exactive HF-X mass spectrometer (Thermo Scientific). Separation of 500ng of peptide mixture was achieved by running a constant flow rate of $250 \mathrm{~nL} / \mathrm{min}$ in $0.1 \%$ formic acid/99.9\% water and a 140 min gradient from $10 \%$ to $95 \%$ ( $23 \%$ for $85 \mathrm{~min}, 38 \%$ for 30 $\min , 60 \%$ for $10 \mathrm{~min}, 95 \%$ for $15 \mathrm{~min}$ ) elution buffer ( $80 \%$ acetonitrile, $0.1 \%$ formic acid, $19.9 \%$ water). A data-dependent acquisition (DDA) method operated under Xcalibur 3.1.66.10 was applied to record the spectra. For the preTAILS and TAILS samples the full scan MS spectra $(350-1500 \mathrm{~m} / \mathrm{z})$ were acquired with a resolution of 120'000 after accumulation to a target value of $3 \mathrm{e} 6$ in profile mode. MS/MS spectra with fixed first mass of $110 \mathrm{~m} / \mathrm{z}$ were recorded in centroid mode from the 12 most intense signals with a resolution of 45,000 applying an automatic gain control of 1e5 and a maximum injection time of $96 \mathrm{~ms}$. Precursors were isolated using a window of $0.7 \mathrm{~m} / \mathrm{z}$ and fragmented with a normalized collision energy (NCE) of $30 \%$. Precursor ions with unassigned, single charge or above charge of six were rejected and precursor masses already selected for MS/MS were excluded for further selection for 60s. Settings for the scan of the secretome peptide mixture were the same except for using a resolution of 17'500 for recording the MS/MS spectra, a maximum injection time of $60 \mathrm{~ms}$, and a NCE of $25 \%$.

Raw files were searched against the Mus musculus database compiled from the UniProt reference proteome (ID: 10090, entries: 17'005) using Sequest from Proteome Discoverer ${ }^{\text {TM }} 2.4$ software (Thermo). The following parameters were selected for database searches: semi-ArgC for enzyme specificity with tolerance of one missed cleavage; carbamidomethyl(C) and TMTpro (K) as fixed modifications, and acetyl(N-term), pyroQ (N-term), TMTpro (N-term), oxidation(M), deamidation (NQ), as variable modifications. Precursor mass error tolerance of 10 
ppm and fragment mass error at 0.02 Da. Percolator was used for decoy control and FDR estimation (0.01 high confidence peptides, 0.05 medium confidence).

For protein-level analysis of the preTAILS sample, quantifiable peptides were summed up per corresponding protein.

Quantifiable non tryptic N-terminal peptides were extracted from the PD analysis of the preTAILS and TAILS samples, normalized based corresponding preTAILS median protein levels. The resulting list was curated for ECM related proteins and were statistically analyzed as it was for the LC-MS/MS data (see details below).

Quantitative Real Time PCR on mouse and human colon tissues

Colon tissues were homogenized using a bead beater homogenizer. Total RNA from was isolated using the PerfectPure RNA Tissue Kit (5 Prime GmbH) according to the manufacturer's protocol. 0.6-1 $\mu \mathrm{g}$ of total RNA is reverse transcribed using High Capacity cDNA Kit (Applied Biosystems inc.). qRT-PCR was performed using SYBR Green PCR Master Mix (Applied Biosystems inc.) on an ABI 7300 instrument (Applied Biosystems). Values are normalized to an Actin- $\beta$ (Actb) control in mouse tissues and to Hypoxanthine-guanine Phosphoribosyltransferase (HPRT) and Ubiquitin C (UBC) controls in the human tissues. Primer sequences are listed in Table S5. Data is presented as mean fold change compared to either healthy WT in mouse tissues or to normal, non-inflamed, human tissues using the $2^{-\Delta \Delta \mathrm{CT}}$ method[68]. Mice samples were grouped according to general state: (i) healthy WT, (ii) pre-symptomatic (day 4 of DSS and healthy IL-10) and (iii) ill (day 10 of DSS and ill IL-10). Standard error of the mean (s.e.m) was calculated on the $2^{-\Delta \mathrm{CT}}$ data, as was the statistical analysis. 
$\underline{\text { Immunohistochemical (IHC) stain for Fibrillin-1 }}$

Tissues were fixed in $4 \%$ paraformaldehyde and embedded in paraffin, and serial $5-\mu \mathrm{m}$ sections were prepared from the whole biopsy for IHC. Sections were de-paraffinized and epitope retrieval was performed. Sections were incubated for $1 \mathrm{~h}$ with anti Fibrillin1 (Abcam), diluted 1:100, at RT followed by secondary antibody (HiDef Detection Polymer, 954D, Cell Marque, Rocklin, CA, USA). A peroxidase substrate kit (SK-4100, Vector Labs, USA) was used as a chromogen and hematoxylin as a counterstain. Tissue exposed only to the secondary antibody was used as negative control.

Staining coverage was quantified as the mean percentage of area covered by staining out of the total image in 3-10 fields of view per sample. The analysis was carried out using ImageJ software (Research Service Branch, NIH) by applying color deconvolution for DAB staining, followed by a consistent binary threshold.

\section{Immunofluorescent (IF) stain for Laminin}

Tissues were embedded in OCT compound, cryosectioned to $10 \mu \mathrm{m}$ and fixed in $4 \%$ paraformaldehyde. Tissue sections were incubated for 60 minutes in blocking solution containing $20 \%$ normal donkey serum and $0.1 \%$ Triton in PBS. Following, sections were incubated for 60 minutes in $5 \mu \mathrm{g} / \mathrm{ml}$ anti-laminin antibody (L9393, Sigma-Aldrich) in a PBS solution containing $2 \%$ normal donkey serum and $0.1 \%$ Triton. Following washes, sections were incubated for 60 minutes with a secondary antibody (Cy3 donkey anti-rabbit) and DRAQ5 nuclear stain. Finally, the slides were washed, mounting solution was added, and they were covered with a coverslip.

Laminin stain sharpness was analyzed by applying a Fourier Transform and measuring the intensity of high frequencies (Fig. S4). These frequencies represent the regions in the image in which edges, i.e. sharp changes in signal intensity, are present, differentiating a diffuse, or 
blurry signal from a sharper one. 2-4 cross sections were analyzed per animal, and for each state (Healthy WT, Day 4 of DSS and Healthy IL-10 $10^{--}$) 4 animals were analyzed.

\section{Quantification and Statistical Analysis}

\section{$\underline{\text { General }}$}

Statistical parameters including the exact value of $n$, the definition of center, dispersion and precision measures (mean \pm s.e.m) and statistical significance are reported and portrayed in the figures and the figure legends. Wherever outlier removal is mentioned, outliers are considered values that are over 3 median absolute deviations distance from the median, which is a robust outlier detection method[69]. Data is judged to be statistically significant when $\mathrm{P}<0.05$ by a two-tailed student's $t$ test or with a Bonferroni correction of $0.05 / \mathrm{m}$, where $\mathrm{m}=$ number of pairwise comparisons. In figures, asterisks denote statistical significance as calculated by student's t test $(*, \mathrm{P}<0.05$ or $0.05 / \mathrm{m} ; * *, \mathrm{P}<0.01$ or $0.01 / \mathrm{m} ; * * *, \mathrm{P}<0.001$ or $0.001 / \mathrm{m})$ in Microsoft Office Excel.

$\underline{\text { Statistical analysis of MS data }}$

Bioinformatics analysis was performed with the Perseus program version 1.5.1.4[70]. The proteomic data was first filtered to remove the potential contaminants, proteins only identified by their modification site and reverse proteins. Next, the intensity values were $\log 2$ transformed and data was filtered to have at least three valid values in each group. Missing values were imputed based on normal distribution and differentially abundant proteins were chosen according to a student's t-test with a Bonferroni correction for multiple (6) pairwise comparisons (corrected $\alpha=0.05 / 6=0.0083$ ), and a 0.05 permutation-based false discovery rate (FDR) cutoff for analysis of multiple proteins[71]. Statistical significance of intersections in Venn diagrams and proportion of differentially abundant ECM proteins out of all differentially 
abundant proteins was assessed using a hypergeometric probability density function in Matlab (Matworks inc.).

\section{Acknowledgments}

We thank Dr. Ori Brenner for assistance in histopathological scoring of intestinal inflammation. Also, we thank Dr. Miriam Rosenberg for helpful comments on the text and styling. Funding: IS is the Incumbent of the Maurizio Pontecorvo Professorial Chair and has received funding from the Israeli Science Foundation (1226/13), European Union's Horizon 2020 research and innovation programme (grant agreement No [801126]), Eu 2020 EDIT consortium , The Thompson Family Foundation, the German-Israeli Foundation for Scientific Research and Development (GIF), the European Research Council AdG (THZCALORIMETRY-DLV695437) and the USA-Israel Binational Science Foundation (712506-01), the Ambach fund, and the Kimmelman center at the WIS. AP thanks the Dept. of Physics of the University of Milano for financial support under the project "Piano di Sviluppo dell'Ateneo per la Ricerca 2014", actions: "A. Upgrade of instrumentation", and "B. Support to young researchers". AS and TG are funded by the Israel Ministry of Science Technology and Space. UadK acknowledges being supported by a Novo Nordisk Foundation Young Investigator Award (NNF16OC0020670) and FS by a H.C. Ørsted COFUND Postdoc Fellowship (COFUNDfellowsDTU, grant agreement no. 713683). IA was supported by the European Molecular Biology Organization (EMBO) Short Term Fellowship [8480].

\section{Author contributions}

Elee Shimshoni: Conceptualization, Methodology, Formal Analysis, Investigation, Writing Original Draft, Writing - Review \& Editing, Visualization, Project administration; Idan Adir: 
Methodology, Investigation, Visualization; Ran Afik: Methodology, Investigation; Inna

Solomonov: Writing - Review \& Editing, Supervision, Project administration; Anjana Shenoy:

Methodology, Formal Analysis; Miri Adler: Formal Analysis, Visualization; Luca Puricelli: Methodology, Formal Analysis, Visualization; Fabio Sabino: Methodology; Simonas Savickas: Methodology; Odelia Mouhadeb: Resources; Nathan Gluck: Resources; Sigal Fishman: Resources; Lael Werner: Resources; Tomer-Meir Salame: Methodology; Dror S. Shouval: Resources, Supervision; Chen Varol: Resources, Supervision; Ulrich auf dem Keller: Methodology, Resources; Alessandro Podestà: Resources, Visualization, Supervision, Funding Acquisition; Tamar Geiger: Resources, Supervision, Funding Acquisition; Paolo Milani: Supervision, Funding Acquisition; Uri Alon: Supervision; Irit Sagi: Conceptualization, Resources, Writing - Review \& Editing, Supervision, Project administration, Funding Acquisition;

\section{Declaration of Interests}

The authors declare no competing interests.

\section{Data availability}

The MS proteomics data have been deposited to the ProteomeXchange Consortium (http://www.proteomexchange.org/) via the PRIDE partner repository with the dataset identifier PXD004740.

\section{References}

[1] M. Grossman, N. Ben-Chetrit, A. Zhuravlev, R. Afik, E. Bassat, I. Solomonov, Y. Yarden, I. Sagi, Tumor cell invasion can be blocked by modulators of collagen fibril alignment that control assembly of the extracellular matrix, Cancer Res. 76 (2016) 4249-4258. https://doi.org/10.1158/0008-5472.CAN-15-2813.

[2] J. Herrera, C.A. Henke, P.B. Bitterman, Extracellular matrix as a driver of progressive fibrosis, J. Clin. Invest. 128 (2018) 45-53. https://doi.org/10.1172/JCI93557.

[3] P. Lu, V.M. Weaver, Z. Werb, The extracellular matrix: A dynamic niche in cancer progression, J. Cell Biol. 196 (2012) 395-406. https://doi.org/10.1083/jcb.201102147. 
[4] R. V Iozzo, M.A. Gubbiotti, Extracellular matrix : The driving force of mammalian diseases, Matrix Biol. 71-72 (2018) 1-9. https://doi.org/10.1016/j.matbio.2018.03.023.

[5] R. Khokha, A. Murthy, A. Weiss, Metalloproteinases and their natural inhibitors in inflammation and immunity, Nat. Rev. Immunol. 13 (2013) 649-665. https://doi.org/10.1038/nri3499.

[6] N.K. Karamanos, A.D. Theocharis, T. Neill, R. V Iozzo, Matrix modeling and remodeling: A biological interplay regulating tissue homeostasis and diseases, Matrix Biol. 75-76 (2019) 1-11. https://doi.org/10.1016/j.matbio.2018.08.007.

[7] E. Shimshoni, D. Yablecovitch, L. Baram, I. Dotan, I. Sagi, ECM remodelling in IBD: innocent bystander or partner in crime? The emerging role of extracellular molecular events in sustaining intestinal inflammation, Gut. 64 (2015) 367-72. https://doi.org/10.1136/gutjnl-2014-308048.

[8] F.E. Castaneda, B. Walia, M. Vijay-Kumar, N.R. Patel, S. Roser, V.L. Kolachala, M. Rojas, L. Wang, G. Oprea, P. Garg, A.T. Gewirtz, J. Roman, D. Merlin, S. V Sitaraman, Targeted deletion of metalloproteinase 9 attenuates experimental colitis in mice: central role of epithelial-derived MMP., Gastroenterology. 129 (2005) 1991-2008. https://doi.org/10.1053/j.gastro.2005.09.017.

[9] P. Garg, M. Vijay-Kumar, L. Wang, A.T. Gewirtz, D. Merlin, S. V Sitaraman, Matrix metalloproteinase-9-mediated tissue injury overrides the protective effect of matrix metalloproteinase-2 during colitis., Am. J. Physiol. Gastrointest. Liver Physiol. 296 (2009) G175-G184. https://doi.org/10.1152/ajpgi.90454.2008.

[10] K. Lohr, H. Sardana, S. Lee, F. Wu, D.L. Huso, A.R. Hamad, S. Chakravarti, Extracellular matrix protein lumican regulates inflammation in a mouse model of colitis, Inflamm. Bowel Dis. 18 (2012) 143-151. https://doi.org/10.1002/ibd.21713.Extracellular.

[11] F.L. Koller, E.A. Dozier, K.T. Nam, M. Swee, T.P. Birkland, W.C. Parks, B. Fingleton, Lack of MMP10 exacerbates experimental colitis and promotes development of inflammation-associated colonic dysplasia., Lab. Invest. 92 (2012) 1749-59. https://doi.org/10.1038/labinvest.2012.141.

[12] M. Waterman, O. Ben-Izhak, R. Eliakim, G. Groisman, I. Vlodavsky, N. Ilan, Heparanase upregulation by colonic epithelium in inflammatory bowel disease., Mod. Pathol. 20 (2007) 8-14. https://doi.org/10.1038/modpathol.3800710.

[13] A. Capuano, E. Pivetta, G. Sartori, G. Bosisio, M. Mongiat, P. Spessotto, Abrogation of EMILIN1- $\mathrm{b} 1$ integrin interaction promotes experimental colitis and colon carcinogenesis, Matrix Biol. 83 (2019) 97-115. https://doi.org/10.1016/j.matbio.2019.08.006.

[14] A.C. Petrey, C.A. De Motte, Hyaluronan in inflammatory bowel disease : Cross-linking inflammation and coagulation, Matrix Biol. 78-79 (2019) 314-323. https://doi.org/10.1016/j.matbio.2018.03.011.

[15] G. Pedersen, T. Saermark, T. Kirkegaard, J. Brynskov, Spontaneous and cytokine induced expression and activity of matrix metalloproteinases in human colonic epithelium, Clin. Exp. Immunol. 155 (2009) 257-265. https://doi.org/10.1111/j.1365-2249.2008.03836.x. 
[16] M.D. Baugh, M.J. Perry, A.P. Hollander, D.R. Davies, S.S. Cross, A.J. Lobo, C.J. Taylor, G.S. Evans, Matrix metalloproteinase levels are elevated in inflammatory bowel disease., Gastroenterology. 117 (1999) 814-822.

[17] M.J.W. Meijer, M.A.C. Mieremet-Ooms, A.M. van der Zon, W. van Duijn, R.A. van Hogezand, C.F.M. Sier, D.W. Hommes, C.B.H.W. Lamers, H.W. Verspaget, Increased mucosal matrix metalloproteinase- $1,-2,-3$ and -9 activity in patients with inflammatory bowel disease and the relation with Crohn's disease phenotype., Dig. Liver Dis. 39 (2007) 733-9. https://doi.org/10.1016/j.dld.2007.05.010.

[18] I. Okayasu, S. Hatakeyama, M. Yamada, T. Ohkusa, Y. Inagaki, R. Nakaya, A novel method in the induction of reliable experimental acute and chronic ulcerative colitis in mice., Gastroenterology. 98 (1990) 694-702.

[19] D. Berg, J. Zhang, J. Weinstock, H. Ismail, Rapid development of colitis in NSAIDtreated IL-10-deficient mice, Gastroenterology. (2002) 1527-1542. https://doi.org/10.1053/gast.rostaglandins.

[20] E.F. Stange, S.P.L. Travis, S. Vermeire, C. Beglinger, L. Kupcinkas, K. Geboes, A. Barakauskiene, V. Villanacci, A. Von Herbay, B.F. Warren, C. Gasche, H. Tilg, S.W. Schreiber, J. Schölmerich, W. Reinisch, European Crohn's and Colitis Organisation, European evidence based consensus on the diagnosis and management of Crohn's disease: definitions and diagnosis., Gut. 55 Suppl 1 (2006)i1-15. https://doi.org/10.1136/gut.2005.081950a.

[21] R. Kühn, J. Löhler, D. Rennick, K. Rajewsky, W. Müller, Interleukin-10-deficient mice develop chronic enterocolitis., Cell. 75 (1993) 263-274. https://doi.org/10.1016/00928674(93)80068-P.

[22] L.M. Keubler, M. Buettner, C. Häger, A. Bleich, A multihit model: Colitis lessons from the interleukin-10-deficient mouse, Inflamm. Bowel Dis. 21 (2015) 1967-1975. https://doi.org/10.1097/MIB.0000000000000468.

[23] E.-O. Glocker, D. Kotlarz, K. Boztug, E.M. Gertz, A.A. Schäffer, F. Noyan, M. Perro, J. Diestelhorst, A. Allroth, D. Murugan, N. Hätscher, D. Pfeifer, K.-W. Sykora, M. Sauer, H. Kreipe, M. Lacher, R. Nustede, C. Woellner, U. Baumann, U. Salzer, S. Koletzko, N. Shah, A.W. Segal, A. Sauerbrey, S. Buderus, S.B. Snapper, B. Grimbacher, C. Klein, Inflammatory bowel disease and mutations affecting the interleukin-10 receptor., N. Engl. J. Med. 361 (2009) 2033-45. https://doi.org/10.1056/NEJMoa0907206.

[24] M. van der Rest, R. Garrone, Collagen family of proteins., FASEB J. (1991).

[25] M.E. McAlindon, T. Gray, A. Galvin, H.F. Sewell, D.K. Podolsky, Y.R. Mahida, Differential lamina propria cell migration via basement membrane pores of inflammatory bowel disease mucosa, Gastroenterology. 115 (1998) 841-848. https://doi.org/10.1016/S0016-5085(98)70255-0.

[26] Y. Fujimura, R. Kamoi, M. Iida, Pathogenesis of aphthoid ulcers in Crohn's disease: Correlative findings by magnifying colonoscopy, electron microscopy, and immunohistochemistry, Gut. 38 (1996) 724-732. https://doi.org/10.1136/gut.38.5.724.

[27] M. Indrieri, A. Podestà, G. Bongiorno, D. Marchesi, P. Milani, Adhesive-free colloidal probes for nanoscale force measurements: production and characterization., Rev. Sci. 
Instrum. 82 (2011) 023708. https://doi.org/10.1063/1.3553499.

[28] L. Puricelli, M. Galluzzi, C. Schulte, A. Podestà, P. Milani, Nanomechanical and topographical imaging of living cells by atomic force microscopy with colloidal probes., Rev. Sci. Instrum. 86 (2015) 033705. https://doi.org/10.1063/1.4915896.

[29] A. Naba, K.R. Clauser, S. Hoersch, H. Liu, S.A. Carr, R.O. Hynes, The Matrisome: In Silico Definition and In Vivo Characterization by Proteomics of Normal and Tumor Extracellular Matrices, Mol. Cell. Proteomics. 11 (2012) M111.014647-M111.014647. https://doi.org/10.1074/mcp.M111.014647.

[30] T.B. Bennike, T.G. Carlsen, T. Ellingsen, O.K. Bonderup, H. Glerup, M. Bøgsted, G. Christiansen, S. Birkelund, A. Stensballe, V. Andersen, Neutrophil Extracellular Traps in Ulcerative Colitis: A Proteome Analysis of Intestinal Biopsies., Inflamm. Bowel Dis. 21 (2015) 2052-67. https://doi.org/10.1097/MIB.0000000000000460.

[31] A.G. Marneros, B.R. Olsen, Physiological role of collagen XVIII and endostatin., FASEB J. 19 (2005) 716-28. https://doi.org/10.1096/fj.04-2134rev.

[32] A. Utriainen, R. Sormunen, M. Kettunen, L.S. Carvalhaes, E. Sajanti, L. Eklund, R. Kauppinen, G.T. Kitten, T. Pihlajaniemi, Structurally altered basement membranes and hydrocephalus in a type XVIII collagen deficient mouse line, Hum. Mol. Genet. 13 (2004) 2089-2099. https://doi.org/10.1093/hmg/ddh213.

[33] R. Heljasvaara, M. Aikio, H. Ruotsalainen, T. Pihlajaniemi, Collagen XVIII in tissue homeostasis and dysregulation - Lessons learned from model organisms and human patients, Matrix Biol. 57-58 (2017) 55-75. https://doi.org/10.1016/j.matbio.2016.10.002.

[34] L.Y. Sakai, D.R. Keene, E. Engvall, Fibrillin, a new 350-kD glycoprotein, is a component of extracellular microfibrils., J. Cell Biol. 103 (1986) 2499-509.

http://www.ncbi.nlm.nih.gov/pubmed/3536967 (accessed July 20, 2017).

[35] F. Ramirez, C. Caescu, E. Wondimu, J. Galatioto, Marfan syndrome ; A connective tissue disease at the crossroads of mechanotransduction, TGF $\beta$ signaling and cell stemness, Matrix Biol. 71-72 (2018) 82-89. https://doi.org/10.1016/j.matbio.2017.07.004.

[36] P.E. Van den Steen, B. Dubois, I. Nelissen, P.M. Rudd, R.A. Dwek, G. Opdenakker, Biochemistry and Molecular Biology of Gelatinase B or Matrix Metalloproteinase-9 (MMP-9), Crit. Rev. Biochem. Mol. Biol. 37 (2002) 375-536. https://doi.org/10.1080/10409230290771546.

[37] Z.-S. Zeng, A.M. Cohen, J.G. Guillem, Loss of basement membrane type IV collagen is associated with increased expression of metalloproteinases 2 and 9 (MMP-2 and MMP-9) during human colorectal tumorigenesis, Carcinogenesis. 20 (1999) 749-755. https://doi.org/10.1093/carcin/20.5.749.

[38] J. Gaffney, I. Solomonov, E. Zehorai, I. Sagi, Multilevel regulation of matrix metalloproteinases in tissue homeostasis indicates their molecular specificity in vivo, Matrix Biol. 44-46 (2015) 191-199. https://doi.org/10.1016/J.MATBIO.2015.01.012.

[39] S.J. George, J.L. Johnson, In Situ Zymography, in: Humana Press, Totowa, NJ, 2010: pp. 271-277. https://doi.org/10.1007/978-1-60327-299-5_17.

[40] A. Kofla-Dlubacz, M. Matusiewicz, M. Krzystek-Korpacka, B. Iwanczak, Correlation of 
MMP-3 and MMP-9 with crohn's disease activity in children, Dig. Dis. Sci. 57 (2012) 706-712. https://doi.org/10.1007/s10620-011-1936-z.

[41] A.M. Howard, K.S. Lafever, A.M. Fenix, C.R. Scurrah, K.S. Lau, D.T. Burnette, G. Bhave, N. Ferrell, A. Page-Mccaw, DSS-induced damage to basement membranes is repaired by matrix replacement and crosslinking, J. Cell Sci. 132 (2019). https://doi.org/10.1242/jcs.226860.

[42] S. Kugathasan, L.A. Denson, T.D. Walters, M.-O. Kim, U.M. Marigorta, M. Schirmer, K. Mondal, C. Liu, A. Griffiths, J.D. Noe, W. V Crandall, S. Snapper, S. Rabizadeh, J.R. Rosh, J.M. Shapiro, S. Guthery, D.R. Mack, R. Kellermayer, M.D. Kappelman, S. Steiner, D.E. Moulton, D. Keljo, S. Cohen, M. Oliva-Hemker, M.B. Heyman, A.R. Otley, S.S. Baker, J.S. Evans, B.S. Kirschner, A.S. Patel, D. Ziring, B.C. Trapnell, F.A. Sylvester, M.C. Stephens, R.N. Baldassano, J.F. Markowitz, J. Cho, R.J. Xavier, C. Huttenhower, B.J. Aronow, G. Gibson, J.S. Hyams, M.C. Dubinsky, Prediction of complicated disease course for children newly diagnosed with Crohn's disease: a multicentre inception cohort study, Lancet. 389 (2017) 1710-1718. https://doi.org/10.1016/S0140-6736(17)30317-3.

[43] F. Rieder, J.R. de Bruyn, B.T. Pham, K. Katsanos, V. Annese, P.D.R. Higgins, F. Magro, I. Dotan, Results of the 4th Scientific Workshop of the ECCO (Group II): Markers of intestinal fibrosis in inflammatory bowel disease., J. Crohns. Colitis. (2014). https://doi.org/10.1016/j.crohns.2014.03.009.

[44] O.H. Nielsen, G. Rogler, D. Hahnloser, O.Ø. Thomsen, Diagnosis and management of fistulizing Crohn's disease., Nat. Clin. Pract. Gastroenterol. Hepatol. 6 (2009) 92-106. https://doi.org/10.1038/ncpgasthep1340.

[45] G. Latella, G. Rogler, G. Bamias, C. Breynaert, J. Florholmen, G. Pellino, S. Reif, S. Speca, I.C. Lawrance, Results of the 4th scientific workshop of the ECCO (I): Pathophysiology of intestinal fibrosis in IBD., J. Crohns. Colitis. (2014). https://doi.org/10.1016/j.crohns.2014.03.008.

[46] F. Rieder, C. Fiocchi, Intestinal fibrosis in IBD - a dynamic , multifactorial process, 6 (2009) 228-235. https://doi.org/10.1038/nrgastro.2009.31.

[47] B.W. Bardoel, E.F. Kenny, G. Sollberger, A. Zychlinsky, The Balancing Act of Neutrophils, Cell Host Microbe. 15 (2014) 526-536. https://doi.org/10.1016/J.CHOM.2014.04.011.

[48] Y. Yan, V. Kolachala, G. Dalmasso, H. Nguyen, H. Laroui, S. V. Sitaraman, D. Merlin, Temporal and Spatial Analysis of Clinical and Molecular Parameters in Dextran Sodium Sulfate Induced Colitis, PLoS One. 4 (2009) e6073. https://doi.org/10.1371/journal.pone.0006073.

[49] D. Talmi-Frank, Z. Altboum, I. Solomonov, Y. Udi, D.A. Jaitin, M. Klepfish, E. David, A. Zhuravlev, H. Keren-Shaul, D.R. Winter, I. Gat-Viks, M. Mandelboim, T. Ziv, I. Amit, I. Sagi, Extracellular Matrix Proteolysis by MT1-MMP Contributes to Influenza-Related Tissue Damage and Mortality., Cell Host Microbe. 20 (2016) 458-470. https://doi.org/10.1016/j.chom.2016.09.005.

[50] D.N.O. Dwyer, S.J. Gurczynski, B.B. Moore, Pulmonary immunity and extracellular matrix interactions, Matrix Biol. 73 (2018) 122-134. 
https://doi.org/10.1016/j.matbio.2018.04.003.

[51] S. Wohlgemuth, D. Haller, M. Blaut, G. Loh, Reduced microbial diversity and high numbers of one single Escherichia coli strain in the intestine of colitic mice, Environ. Microbiol. 11 (2009) 1562-1571. https://doi.org/10.1111/j.1462-2920.2009.01883.x.

[52] N.M.J. Schwerbrock, M.K. Makkink, M. van der Sluis, H.A. Büller, A.W.C. Einerhand, R.B. Sartor, J. Dekker, Interleukin 10-deficient mice exhibit defective colonic Muc2 synthesis before and after induction of colitis by commensal bacteria, Inflamm. Bowel Dis. 10 (2004) 811-823. https://doi.org/10.1097/00054725-200411000-00016.

[53] C. Becker, M.C. Fantini, M.F. Neurath, High resolution colonoscopy in live mice., Nat. Protoc. 1 (2006) 2900-2904. https://doi.org/10.1038/nprot.2006.446.

[54] E. Zigmond, C. Varol, J. Farache, E. Elmaliah, A.T. Satpathy, G. Friedlander, M. Mack, N. Shpigel, I.G. Boneca, K.M. Murphy, G. Shakhar, Z. Halpern, S. Jung, Ly6C hi Monocytes in the Inflamed Colon Give Rise to Proinflammatory Effector Cells and Migratory Antigen-Presenting Cells, Immunity. (2012). https://doi.org/10.1016/j.immuni.2012.08.026.

[55] G.K. Behbehani, C. Thom, E.R. Zunder, R. Finck, B. Gaudilliere, G.K. Fragiadakis, W.J. Fantl, G.P. Nolan, Transient partial permeabilization with saponin enables cellular barcoding prior to surface marker staining, Cytometry. A. 85 (2014) 1011. https://doi.org/10.1002/CYTO.A.22573.

[56] E.R. Zunder, R. Finck, G.K. Behbehani, E.D. Amir, S. Krishnaswamy, V.D. Gonzalez, C.G. Lorang, Z. Bjornson, M.H. Spitzer, B. Bodenmiller, W.J. Fantl, D. Pe'er, G.P. Nolan, Palladium-based mass tag cell barcoding with a doublet-filtering scheme and single-cell deconvolution algorithm, Nat. Protoc. 10 (2015) 316-333. https://doi.org/10.1038/nprot.2015.020.

[57] H. Lu, T. Hoshiba, N. Kawazoe, G. Chen, Comparison of decellularization techniques for preparation of extracellular matrix scaffolds derived from three-dimensional cell culture., J. Biomed. Mater. Res. A. 100 (2012) 2507-16. https://doi.org/10.1002/jbm.a.34150.

[58] E. Shimshoni, I. Sagi, Sample Preparation of Extracellular Matrix of Murine Colons for Scanning Electron Microscopy, in: I. Sagi, N. Afratis (Eds.), Collagen. Methods Mol. Biol., Humana Press, New York, NY, 2019: pp. 129-133. https://doi.org/10.1007/978-14939-9095-5_9.

[59] H.-J. Butt, B. Cappella, M. Kappl, Force measurements with the atomic force microscope: Technique, interpretation and applications, Surf. Sci. Rep. 59 (2005) 1-152. https://doi.org/10.1016/j.surfrep.2005.08.003.

[60] H. Schillers, C. Rianna, J. Schäpe, T. Luque, H. Doschke, M. Wälte, J.J. Uriarte, N. Campillo, G.P.A. Michanetzis, J. Bobrowska, A. Dumitru, E.T. Herruzo, S. Bovio, P. Parot, M. Galluzzi, A. Podestà, L. Puricelli, S. Scheuring, Y. Missirlis, R. Garcia, M. Odorico, J.-M. Teulon, F. Lafont, M. Lekka, F. Rico, A. Rigato, J.-L. Pellequer, H. Oberleithner, D. Navajas, M. Radmacher, Standardized Nanomechanical Atomic Force Microscopy Procedure (SNAP) for Measuring Soft and Biological Samples, Sci. Rep. 7 (2017) 5117. https://doi.org/10.1038/s41598-017-05383-0.

[61] H. Cramér, Mathematical Methods of Statistics, Princet. Univ. Press. (1999). 
[62] J. Cox, M. Mann, MaxQuant enables high peptide identification rates, individualized p.p.b.-range mass accuracies and proteome-wide protein quantification., Nat. Biotechnol. 26 (2008) 1367-1372. https://doi.org/10.1038/nbt.1511.

[63] S. Tyanova, T. Temu, J. Cox, The MaxQuant computational platform for mass spectrometry - based shotgun proteomics, Nat. Protoc. 11 (2016) 2301-2319. https://doi.org/10.1038/nprot.2016.136.

[64] J. Cox, N. Neuhauser, A. Michalski, R. a. Scheltema, J. V. Olsen, M. Mann, Andromeda: A peptide search engine integrated into the MaxQuant environment, J. Proteome Res. 10 (2011) 1794-1805. https://doi.org/10.1021/pr101065j.

[65] J. Cox, M.Y. Hein, C.A. Luber, I. Paron, N. Nagaraj, M. Mann, Accurate Proteome-wide Label-free Quantification by Delayed Normalization and Maximal Peptide Ratio Extraction, Termed MaxLFQ, Mol. Cell. Proteomics. 13 (2014) 2513-2526. https://doi.org/10.1074/mcp.M113.031591.

[66] S.L. Salzberg, C4.5: Programs for Machine Learning by J. Ross Quinlan. Morgan Kaufmann Publishers, Inc., 1993, Mach. Learn. 16 (1994) 235-240. https://doi.org/10.1007/BF00993309.

[67] E. Madzharova, F. Sabino, U. auf dem Keller, Exploring extracellular matrix degradomes by TMT-TAILS N-terminomics, in: Methods Mol. Biol., Humana Press Inc., 2019: pp. 115-126. https://doi.org/10.1007/978-1-4939-9095-5_8.

[68] K.J. Livak, T.D. Schmittgen, Analysis of relative gene expression data using real-time quantitative PCR and the 2(- Delta Delta Ct) Method, Methods. 25 (2001) 402-408. https://doi.org/10.1006/meth.2001.1262.

[69] C. Leys, C. Ley, O. Klein, P. Bernard, L. Licata, Detecting outliers : Do not use standard deviation around the mean, use absolute deviation around the median, J. Exp. Soc. Psychol. (2013) 4-6.

[70] S. Tyanova, T. Temu, P. Sinitcyn, A. Carlson, M.Y. Hein, T. Geiger, M. Mann, J. Cox, The Perseus computational platform for comprehensive analysis of (prote)omics data, Nat. Methods. 13 (2016) 731-740. https://doi.org/10.1038/nmeth.3901.

[71] V.G. Tusher, R. Tibshirani, G. Chu, Significance analysis of microarrays applied to the ionizing radiation response, Proc. Natl. Acad. Sci. 98 (2001) 5116-5121. https://doi.org/10.1073/pnas.091062498.

[72] I. Solomonov, E. Zehorai, D. Talmi-Frank, S.G. Wolf, A. Shainskaya, A. Zhuravlev, E. Kartvelishvily, R. Visse, Y. Levin, N. Kampf, D.A. Jaitin, E. David, I. Amit, H. Nagase, I. Sagi, Distinct biological events generated by ECM proteolysis by two homologous collagenases., Proc. Natl. Acad. Sci. U. S. A. 113 (2016) 10884-9. https://doi.org/10.1073/pnas.1519676113. 
Figure 1 Definition of the disease course and tissue states in the two murine colitis models (A) Acute model - DSS-induced colitis. Clinical and endoscopic symptoms are apparent from day 7 and peak at day 10. Chronic model - PAC IL-10 $0^{-/-}$model. Clinical and endoscopic symptoms develop over the course of 14 days, and the chronic inflammatory state is persistent per mouse. Chronically ill IL- $10^{-/-}$mice were harvested at least two weeks following piroxicam discontinuation. (B) H\&E-stained colonic sections of mice from the two models at the indicated states. Note that immune cell infiltration and mucosal damage is not evident on day 4 of the acute model, but is substantial on day 10 . In the PAC IL-10 $0^{-1-}$ model large amounts of immune cell infiltrate is observed in the mucosa (indicated by arrow), but is absent in the healthy IL-10 $10^{-1-}$ mouse. (C) Quantification of the percentage of mice in each clinical category according to endoscopic evaluation (0-4: "healthy"; 5-7 "mildly inflamed"; 8-11 "inflamed"; and 12-15 "severely inflamed", see Fig. S1 and Experimental procedures for details) at the indicated time points or states of both models $(\mathrm{n}>30)$. Note that some $\mathrm{IL}-10^{-/-}$mice develop spontaneous inflammation without piroxicam exposure, and that some do not develop inflammatory symptoms following exposure. Only mice evaluated as "healthy" were used for further analysis as the day 4 of DSS or healthy IL- $10^{-/-}$states. (D) Histological scoring of H\&E stained sections, such as the ones shown in B. Details on scoring are available in the Experimental procedures. In the DSS model, a significant increase in histopathological score was only observed on day 10 and not on day 4 (NS=not significant). Chronically ill $\mathrm{IL}-10^{-/-}$mice display a significant increase in histopathological score. The plot displays the scores of individual animals and the bars represent the state's mean \pm s.e.m. Statistical significance was determined using a student's t-test with a Bonferroni correction for two comparisons to healthy WT for the DSS model (NS: P> $0.025, * * * \mathrm{P}<0.0005)$ and with no correction, compared to healthy IL-10 $0^{-/-}$in the PAC IL-10 $0^{-/-}$ model (*** $\mathrm{P}<0.001)$. (E) Mouse body weight changes over the course of the two models, presented as percentage from body weight on day 0. Significant weight loss in the DSS model appears from day 5 compared to day 2 . Number of animals: $\mathrm{n}(2)=85, \mathrm{n}(4)=55, \mathrm{n}(5)=36$, $\mathrm{n}(7)=41, \mathrm{n}(8)=10, \mathrm{n}(10)=52$; Bonferroni for five comparisons: $* * * \mathrm{P}<0.0002$. Significant weight loss appears in the IL-10 $10^{--}$mice from day 5 compared to day 1 . Number of animals: $\mathrm{n}(1)=8$, $\mathrm{n}(5)=22, \mathrm{n}(7)=7, \mathrm{n}(9)=7, \mathrm{n}(10)=13, \mathrm{n}(12)=19, \mathrm{n}(14)=10, \mathrm{n}(17)=10, \mathrm{n}(21)=17$; Bonferroni correction for eight comparisons: $* \mathrm{P}<0.00625, * * \mathrm{P}<0.00125, * * * \mathrm{P}<1.25 \mathrm{X} 10^{-4}$. Note that mice regain their weight as they reach the chronic phase of colitis.

\section{Figure 2 The ECM suffers structural damage and softening during colitis}

(A) Second-harmonic imaging of mouse colon on the indicated time points over the course of the disease, corresponding to distinct states: healthy WT, pre-symptomatic (day 4 of DSS and healthy IL-10 $0^{--}$), acute inflammation (day 10 of DSS) and chronic inflammation (Chronically ill IL-10 ${ }^{-1-}$ ). Vast ECM structural changes occur in acute inflammation, as indicated in the comparison between day 0 and day 10 . Remarkably, some reorganization of the ECM is apparent from day 4, before clinical symptoms are evident. Healthy IL- $10^{-/-}$display different ECM structure than that of WT mice, with ECM condensation similar to that of day 4. Chronic illness leads to overall maintained crypt architecture, but with loosely packed ECM. (B) SEM images of decellularized colonic ECM at the corresponding states, at three different magnifications. Note the damage and crypt size heterogeneity apparent on day 10 compared to Healthy WT colon. Also note, that the ECM under chronic illness is characterized by perforated crypt walls with thin fibrils. Most remarkably, the two pre-symptomatic states show a common feature of exposure of fibrillar ECM proteins on the crypt walls, as can be observed in the X50k magnification 
(examples are indicated by arrows). (C) Measurement of crypt diameter and distance between adjacent crypt edges in second-harmonic images on all five states - healthy WT (green), DSS day 4 (orange), DSS day 10 (red), healthy IL-10 $0^{-/-}$(yellow) and ill IL-10 $0^{-/-}$(pink). In oval crypts, the largest diameter was always chosen, and the shortest distance was taken for distance measurements. Note that each state has a different mean diameter and distance. Changes in the standard deviations (STDs) of these measures per animal are also apparent, demonstrating their heterogeneity. Both of these measures indicate the architectural changes taking place in the ECM. Statistical significance was determined using a t-test with a Bonferroni correction for five comparisons, and is indicated by asterisks. $* \mathrm{P}<0.01$, $* * \mathrm{P}<0.002$, $* * * \mathrm{P}<0.0002$. The bars represent the mean \pm s.e.m of the state, while each dot corresponds to the mean/standard deviation of one animal, which is based on the analysis of 2-8 $440 \mu \mathrm{mX} 440 \mu \mathrm{m}$ images capturing $\mathrm{n}>30$ crypts per animal. One outlier was removed in the day 4 group. (D) The relative frequency of exposed fibrils on crypt walls in the SEM images, indicating a damaged ECM underlying the epithelium, in Healthy WT vs. Day 4 of DSS and Healthy IL- $10^{-1}$ samples. $* * \mathrm{P}<0.005$, $* * * \mathrm{P}<0.0005, \mathrm{n}=4$ different samples per state, based on analysis of $\mathrm{n}>14$ crypts. (E) ECM softening during colitis progression: individual dots represent the properly weighted median values of the Young's modulus for each animal and the bars represent the average median \pm STD, corresponding to all distributions represented in Fig. S3. There is a significant reduction in ECM rigidity between the healthy WT and acutely inflamed samples as denoted by the asterisks (according to a t-test with Bonferroni correction for three comparisons, ${ }^{*} \mathrm{P}<0.017$ ). The differences between the pairs HC-D4 and D4-D10 are not statistically significant ( $\mathrm{P}=0.30$ and $\mathrm{P}=0.09$, respectively); nevertheless, pre-symptomatic ECMs (D4) show an intermediate behavior with respect to the healthy and acutely inflamed cases. Also, ECM rigidity significantly reduces as IL- $10^{-/-}$mice become chronically ill, as denoted by the asterisks $(* * \mathrm{P}<0.01)$.

\section{Figure 3 Pre-symptomatic states converge to a unique proteomic signature}

(A) Scheme showing the number of differentially abundant ECM proteins, between pairs in the five different states, represented by lines stretched between two nodes of two states; $n$ (Healthy $\mathrm{WT})=\mathrm{n}($ Day 4$)=\mathrm{n}($ Healthy $\mathrm{IL}-10)=8, \mathrm{n}($ Day 10$)=\mathrm{n}(\mathrm{Ill} \mathrm{IL}-10)=10$. Note that the two presymptomatic states are similar in terms of ECM composition. Statistical significance of amount of ECM proteins changing among all ECM proteins and all differentially abundant proteins, according to the sum of hypergeometric probabilities for achieving each number or above it, is indicated next to each comparison by asterisks. $* * \mathrm{P}<0.01, * * * \mathrm{P}<0.001, \mathrm{NS}=$ not significant. $(\mathrm{B}+\mathrm{C})$ Venn diagrams presenting the differentially abundant ECM proteins in the colon comparing healthy WT vs. the pre-symptomatic day 4 of the DSS model (pink) and healthy WT vs. pre-symptomatic healthy IL-10 $0^{-/-}$(blue) in B; and comparing pre-symptomatic day 4 of the DSS model vs. acutely ill day 10 of the DSS model (pink) and pre-symptomatic healthy IL-10 ${ }^{-/-}$ vs. chronically ill IL-10 $0^{-/-}$(blue) in C. The direction of change in abundance in pre-symptomatic states is indicated by arrows. Note the differentially abundant proteins shared among the two comparisons (purple) in both diagrams. The intersection between the differentially abundant proteins in both comparisons is statistically significant ( $p=1.0 \times 10-4$ in $B$ and $p=1.2 \times 10-5$ in $C$ ). Statistical significance in this figure was calculated by the sum of hypergeometric probabilities for achieving each number or above it. (D) MS data of 110(ECM proteins) $\mathrm{x} 44$ (samples at five clinical states) was clustered according to similar protein abundance by computing the squared Euclidean distance. Following, the data was partitioned into three clusters. Grouping according to clinical state is indicated on the $\mathrm{x}$ axis. Protein abundances were normalized to the mean of 
each protein. (E) PCA on the 3(clusters) x 44(samples) data after taking the mean of each cluster. The first PC explains more than $86 \%$ of the variance in the data. PC1 mostly $(\sim 75 \%)$ consists of cluster 1 . The PC1 values for each state are presented in a box plot. $* \mathrm{P}<0.05, * * \mathrm{P}<0.01$, $* * * \mathrm{P}<0.001$. Note that the results show that ECM composition of clinical states can be projected onto a healthy-acutely ill axis, while highlighting the existence of the pre-symptomatic state. Figure 4 Identification of potential ECM biomarkers via machine learning and human validation

(A) Significantly different ECM proteins from MS analysis display similar trends in human dataset. Unpublished data from a proteomic data set of colon biopsies, Bennike et al.[30] were analyzed for changes in ECM protein abundance, comparing UC[30] patients to healthy controls. Proteins that changed significantly between healthy (WT or IL-10-/-) and ill (day 10 of DSS or chronically ill IL-10-/-, respectively) mice were chosen. (B) The directions of the observed changes in human were compared to that of the significant changes in mouse. Proteins that change in the same direction in both species were counted as those that "Agree" with the mouse data, compared to those that "Disagree". A chi-square test of goodness-of-fit was performed to determine whether the rate of agreement in the direction of change was likely to occur by chance by comparing to a 50\% (22.5) "Agree"-50\% (22.5) "Disagree" distribution. (C) A method for identifying potential biomarkers by applying a classification algorithm. Samples were divided into Healthy WT, Pre-symptomatic (day 4 of DSS and healthy IL-10-/-) and Ill (day 10 DSS and ill IL-10-/-), and a J48 decision-tree classification algorithm [33] was applied to the data, along with a stratified 10-fold cross-validation. The two proteins chosen for the tree nodes (Col18a1 and Fbn1) are those that their abundance separate the three groups with the highest accuracy (90.9\%) of all proteins. Col18a1 threshold: $26.67 \log 2$ (LFQ intensity). Fbn1 threshold: 28.76 $\log 2$ (LFQ intensity). (D) Plot of all samples according only to the abundances of Col18a1 and Fbn1, demonstrating that the levels of the two proteins are good indicators of the tissue state. (E) Gene expression analysis of Collagen XVIII (Col18a1) and Fibrillin-1 (Fbn1) on independent mouse tissue samples of all three groups. Note, that Col18a1 transcription is significantly elevated in ill samples, while no significant changes were observed in Fbn1. n(Healthy WT)=5, $\mathrm{n}($ Pre-symptomatic) $=10, \mathrm{n}(\mathrm{Ii1})=9$ (one outlier removed); $* \mathrm{P}<0.0167$, t-test with Bonferroni correction. (F) Validation of biomarker transcript levels on an independent set of human samples. Biopsies and surgical resections of healthy donors or of inflamed colonic mucosa of IBD patients were analyzed for mRNA expression levels of COL18A1 and FBN1. Both predicted markers indicated by the classification model performed on the murine models display significantly elevated levels of 6.2 fold (COL18A1) and 5.5 fold (FBN1) in inflamed IBD tissue compared to tissue from healthy donors $(\mathrm{n}(\mathrm{IBD})=10, \mathrm{n}($ Healthy $)=11 ; * \mathrm{P}<0.05, * * \mathrm{P}<0.01) .(\mathrm{G})$ FBN1 IHC staining in rectal biopsies of healthy donors vs. inflamed regions in UC patients. Controls do not demonstrate FBN1 staining (FBN1 negative), while a subset ( $n=5)$ of UC patients display a strong epithelial staining (FBN1 positive). (H) Quantification of the area covered by FBN1 staining. The distribution of the FBN1 coverage in UC patients is broad, and half of the patients analyzed are FBN1 positive (above 10\% coverage), such as the one presented in $\mathrm{G}$.

Figure 5 Pre-symptomatic tissues display elevated gelatinase expression and activity, and Laminin degradation

(A) Gene expression analysis of selected ECM remodeling enzymes (Mmp-2/-7/-9/-14) and inhibitor (TIMP1) at pre-symptomatic states. An independent set of mouse tissue samples of 
Healthy WT (green), Day 4 of DSS (orange) and Healthy IL-10-/- (yellow) were used. Note, that gelatinases (Mmp-2/-9) are significantly upregulated at pre-symptomatic states. $n=5$ animals per state; $* \mathrm{P}<0.025, * * \mathrm{P}<0.005$, t-test with Bonferroni correction. (B) In situ zymography with DQ GelatinTM on colon cross-sections, depicting elevated gelatinase activity (green) in the mucosa (possibly the epithelium) of the pre-symptomatic states, along with DRAQ5-stained cell nuclei (blue) and SHG signal of fibrillary collagen (red). (C) Quantification of signal intensity in the mucosa, revealing that the elevation in gelatinase activity is statistically significant. (D) Immunofluorescent staining for Laminin, comparing Healthy WT to the two pre-symptomatic states, reveals the pre-symptomatic states are characterized by a more diffused appearance of the protein distribution. (E) Quantification of the diffuse appearance by measuring the intensity of high frequencies of a Fourier Transform applied to the mucosal regions. $n=4$ animals per state; $* \mathrm{P}<0.025$, t-test with Bonferroni correction (see Fig. S4 for details). (F) Degradomic analysis comparing Healthy WT (green) to Day 4 of DSS (orange) reveals elevated degradation of Laminin, Collagen VI and Vitronectin. $\mathrm{n}=4$ animals per state.

Figure 6 Enzyme-bearing immune cells participate in pre-symptomatic ECM remodeling (A) tSNE analysis of mass cytometry demonstrating the differences in cell composition comparing healthy WT mice to the pre-symptomatic states. Each plot represents a pool of eightnine animals analyzed per state and each point corresponds to one cell. Note that monocytes and neutrophils are elevated at day 4 of DSS, while in healthy IL-10-/- mostly adaptive immune system cells are increased compared to WT. (B-G) Scatter dot plots based on the mass cytometry analysis depicting the changes in the relative frequencies (out of all live cells) of the indicated cell populations positive for specific ECM remodeling enzymes at each state: healthy WT (green), day 4 of DSS (orange), healthy IL-10-/-(yellow). Note that the different immune cells carry a variety of enzymes, including the gelatinases, MMP-2 and MMP-9. Additional data from this analysis appears in Fig. S5. Number of animals: $n=9$ for healthy IL-10-/- and $n=8$ for healthy WT and day 4 of DSS (one outlier removed for each group); Significant changes indicated are compared to healthy WT. Bonferroni correction for two comparisons $(* \mathrm{P}<0.025, * * \mathrm{P}<0.005)$.

\section{Scheme 1 Pathophysiological contributions of the pre-symptomatic ECM} The scheme demonstrates how the ECM signatures found at the pre-symptomatic state contribute to the inflammatory symptoms found in colitis. (A) Pre-symptomatic colon segment along with its vasculature. (B) Epithelial crypts composing the colonic mucosa. (C) Subclinical immune cell infiltrate provides easy access for additional immune cell infiltration via MMPs. (D) Gelatinase activity creates an injured basement membrane, thus compromising mucosal barrier function, which grants gut microbes access to the internal environment. (E) Dual effect of increased MMP and LOX secretion along with increased fibrillary collagen secretion. This creates a deformed morphology characterized by both thick fibrous walls circumscribing the crypt in the presence of a perforated basement membrane. 
Figure 1

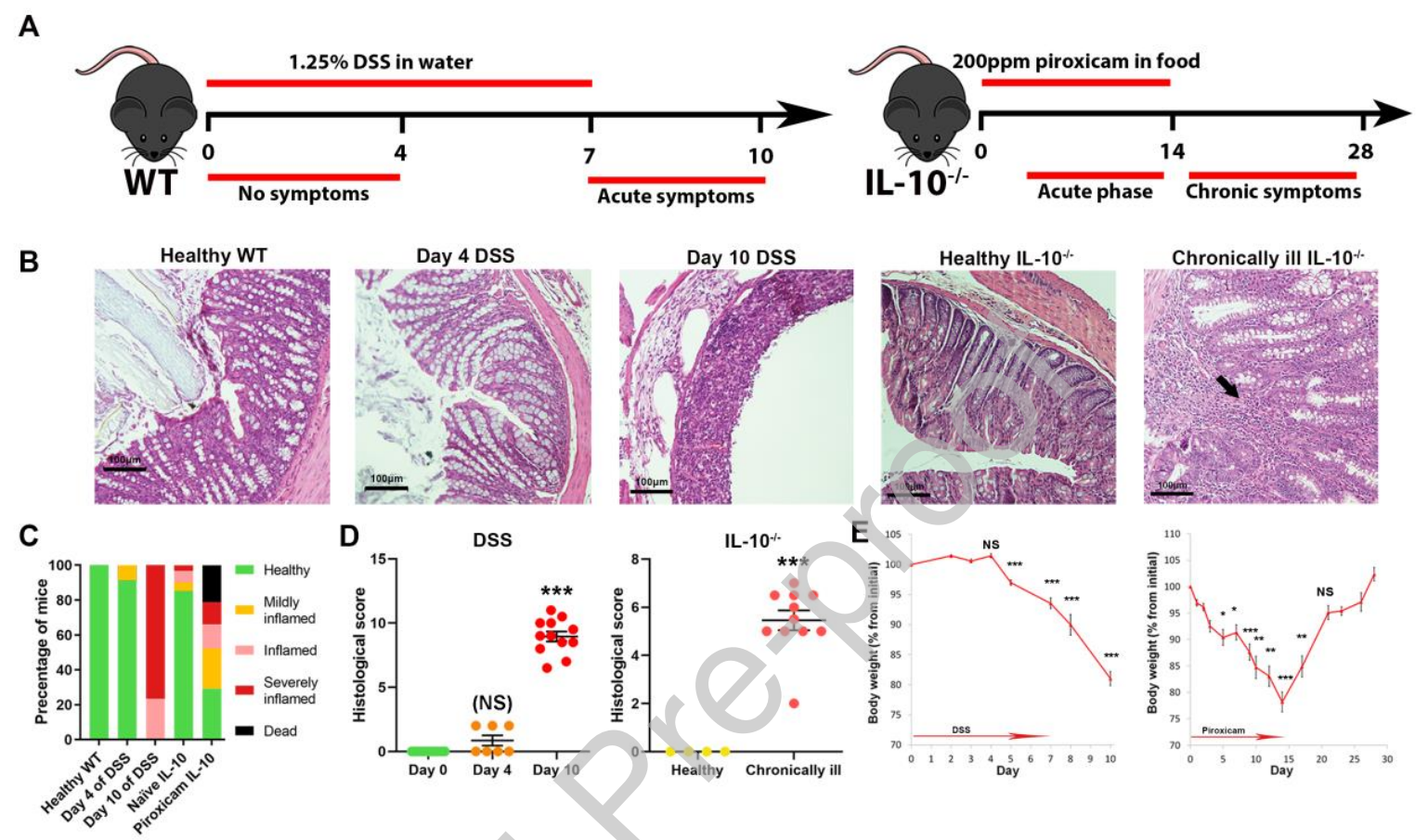


Figure 2
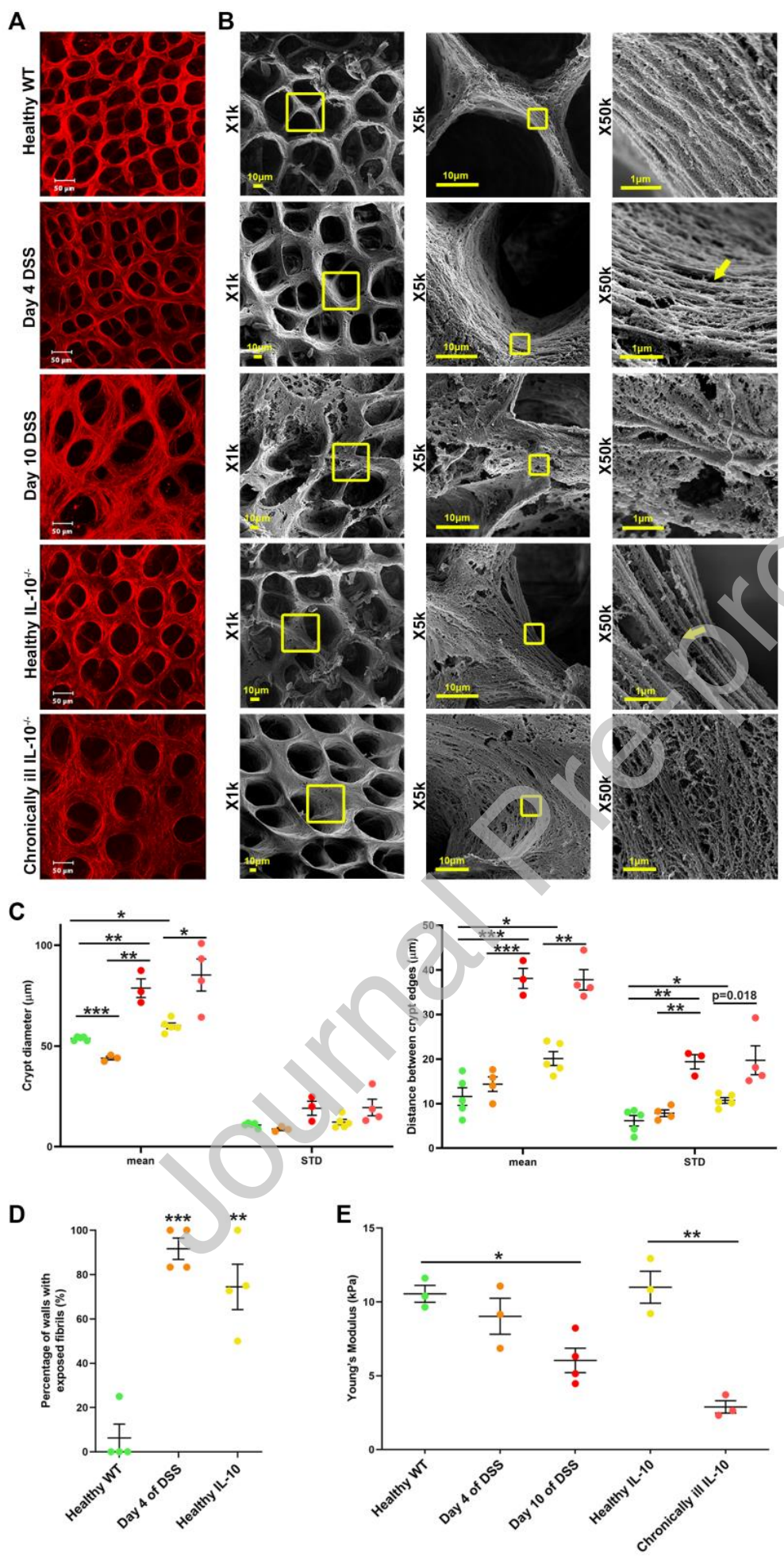


\section{Figure 3}

A

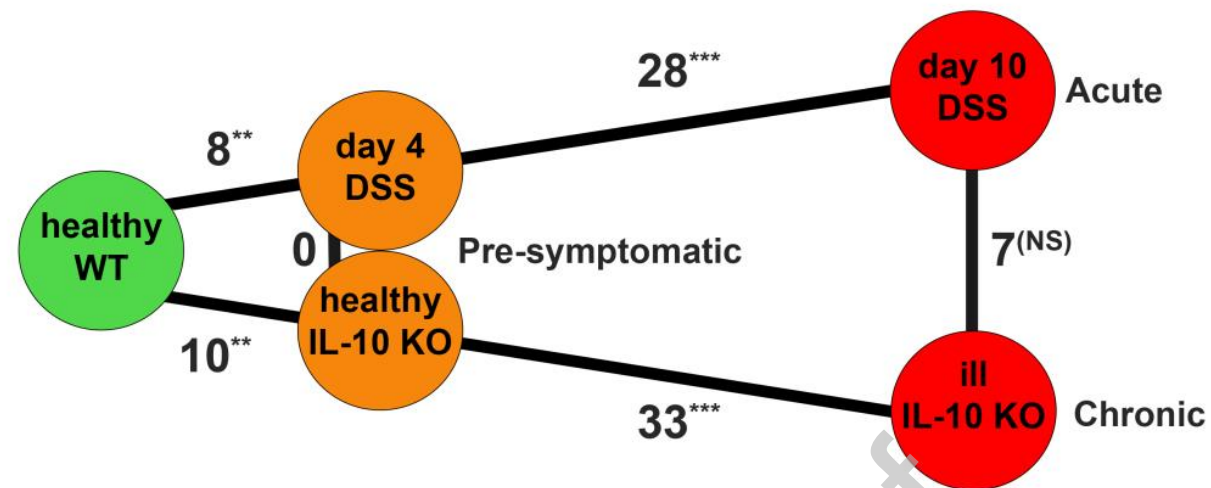

(numbers represent ECM proteins that differ in abundance)

B

Pre-symptomatic vs. Healthy WT

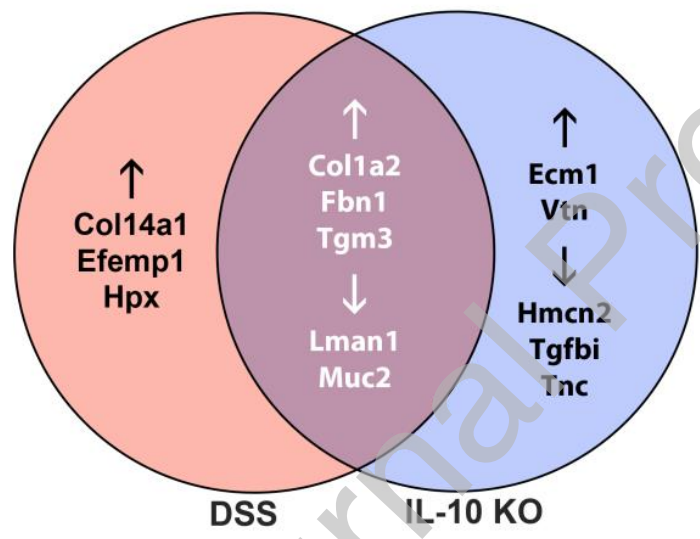

D

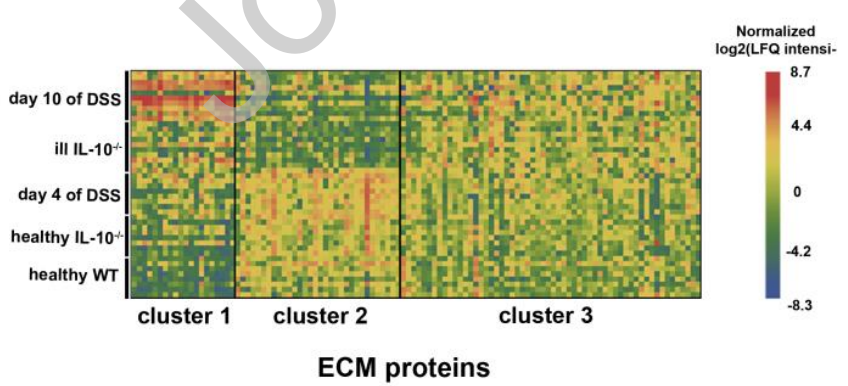

C

Pre-symptomatic vs. Illness

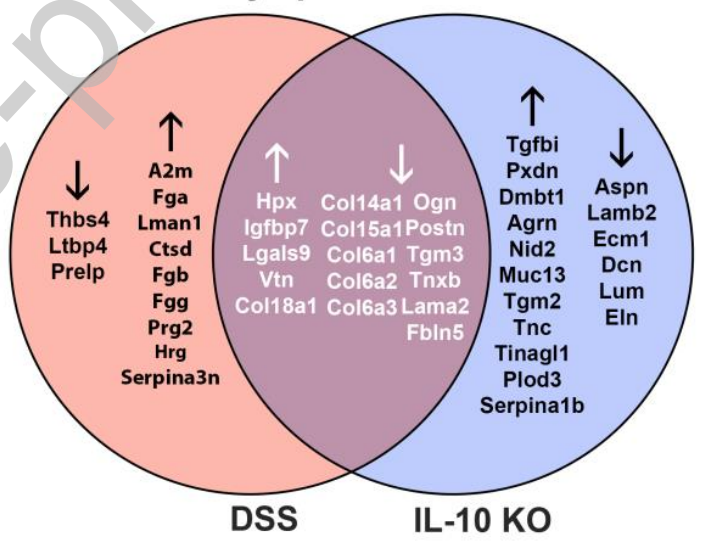

E

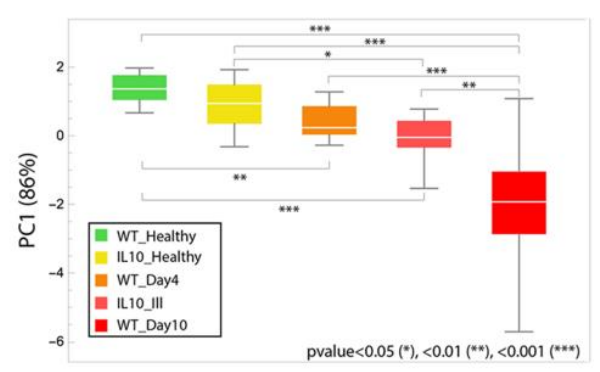


Figure 4

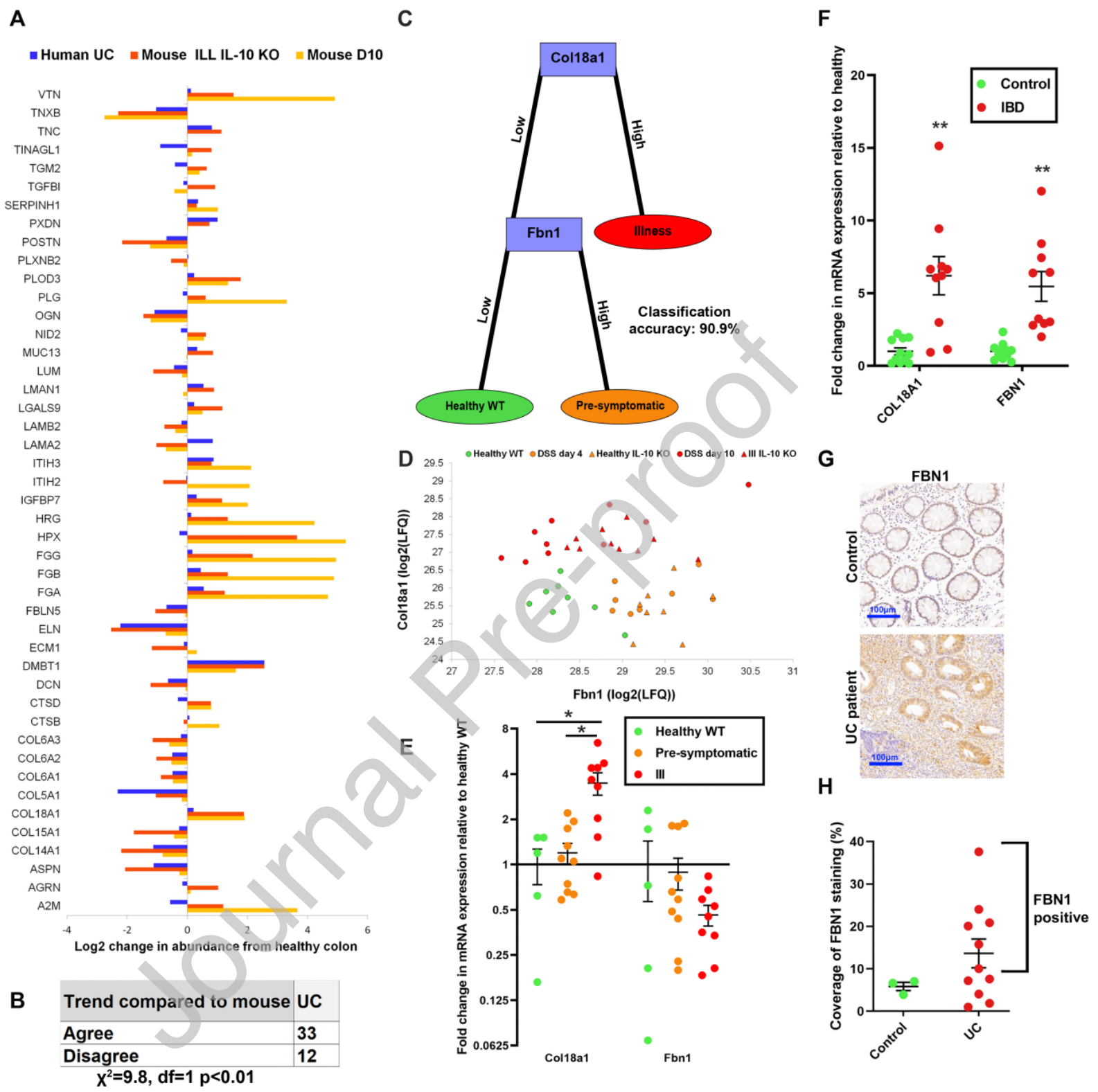


Figure 5

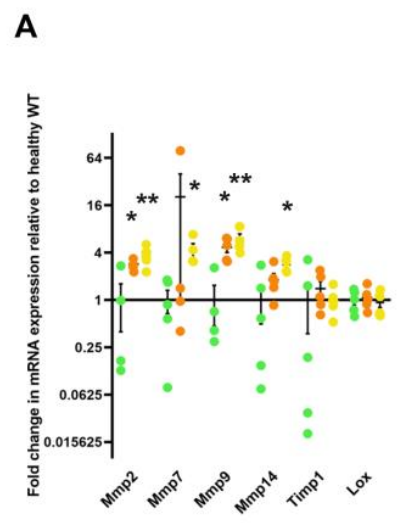

B
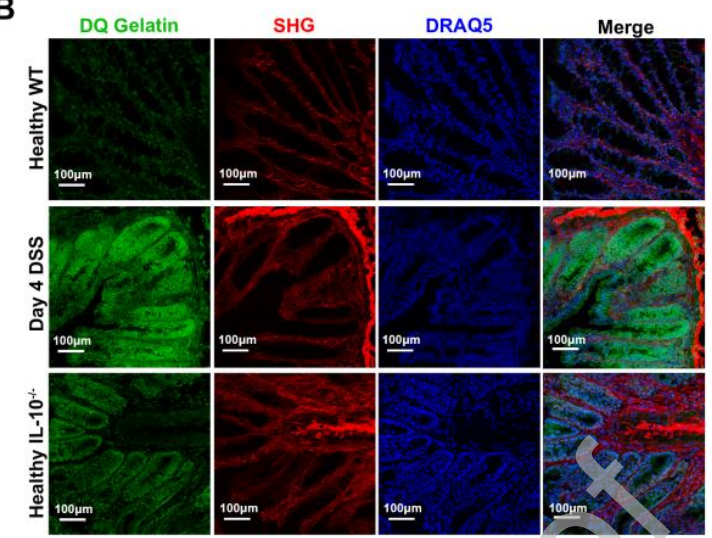

D

5

Laminin

DRAQ5

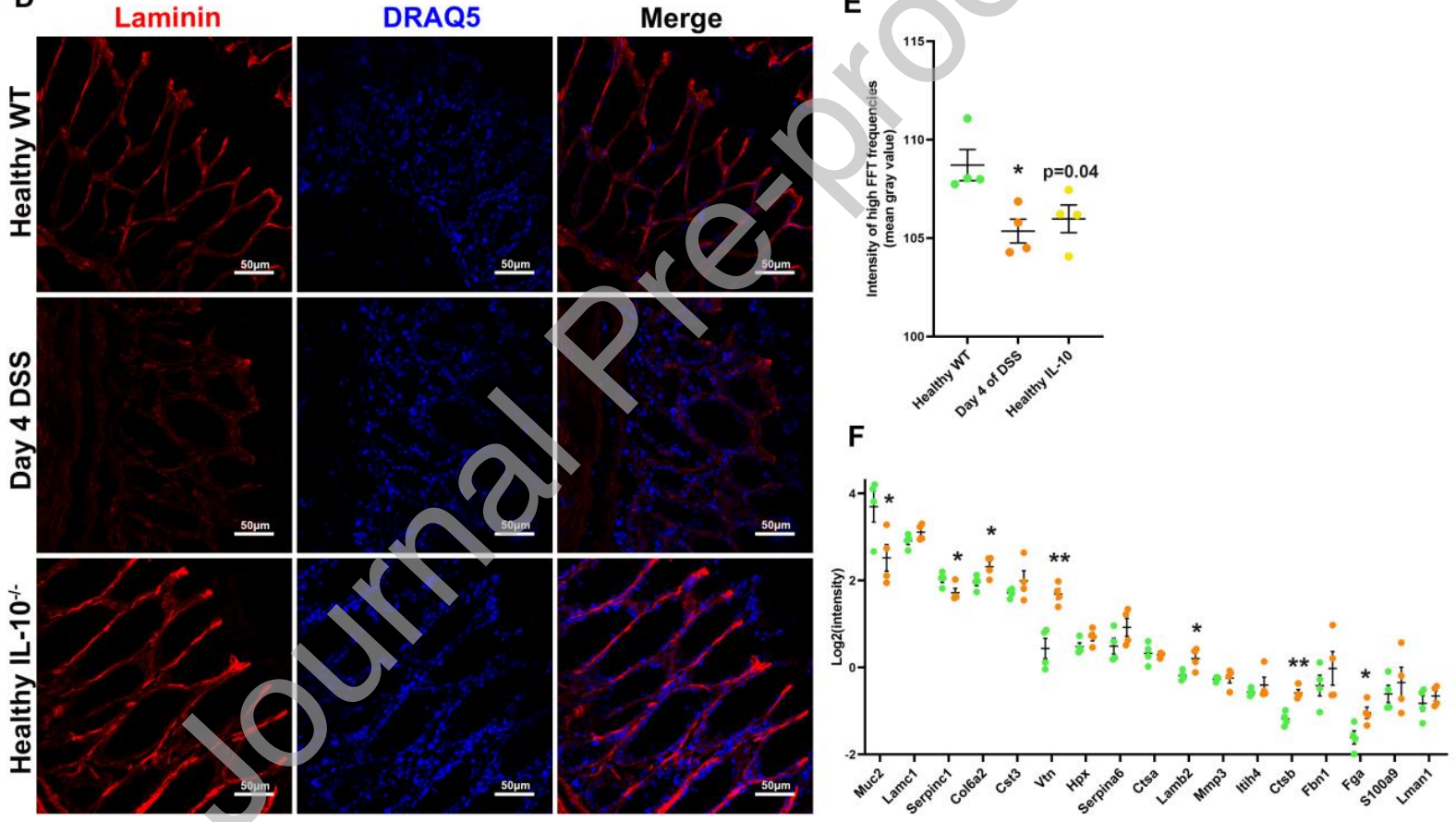

E

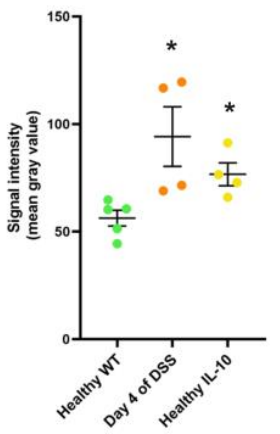




\section{Figure 6}
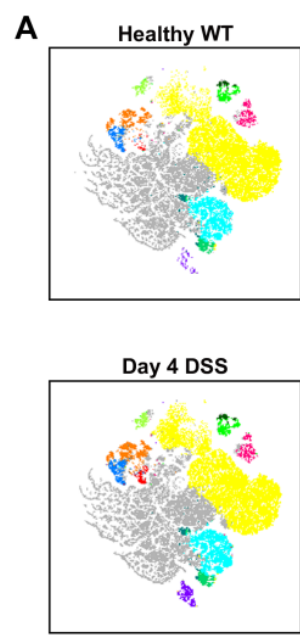

Healthy IL-10

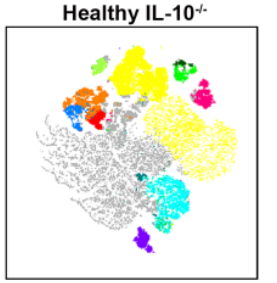

B

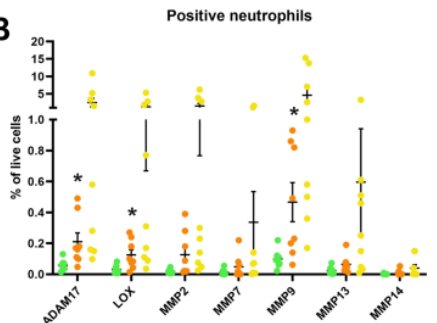

C

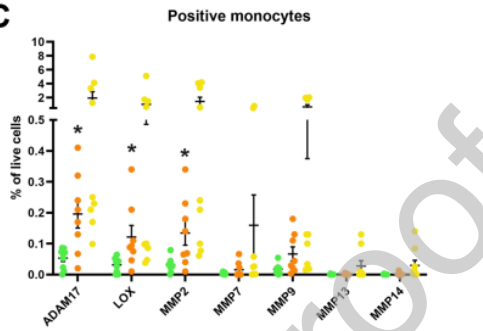

Epithelial

Adult endothelia cells

Late endothelia progenitors

Early endothelia progenitors

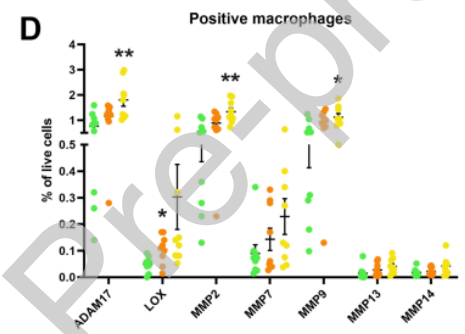

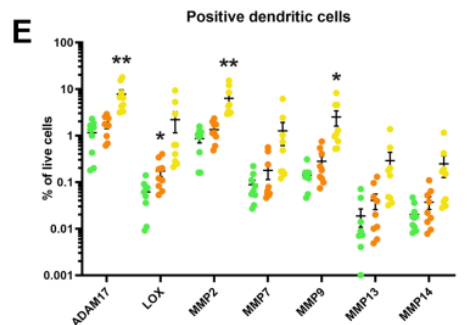

$\mathbf{F}$

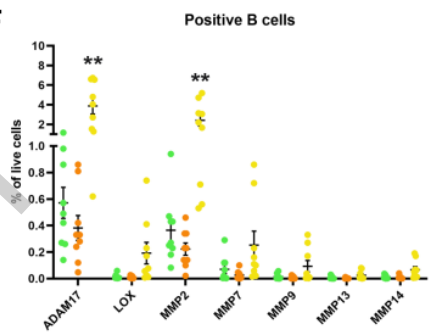

G

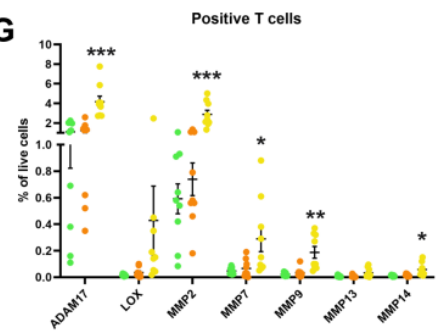




\section{Scheme 1}

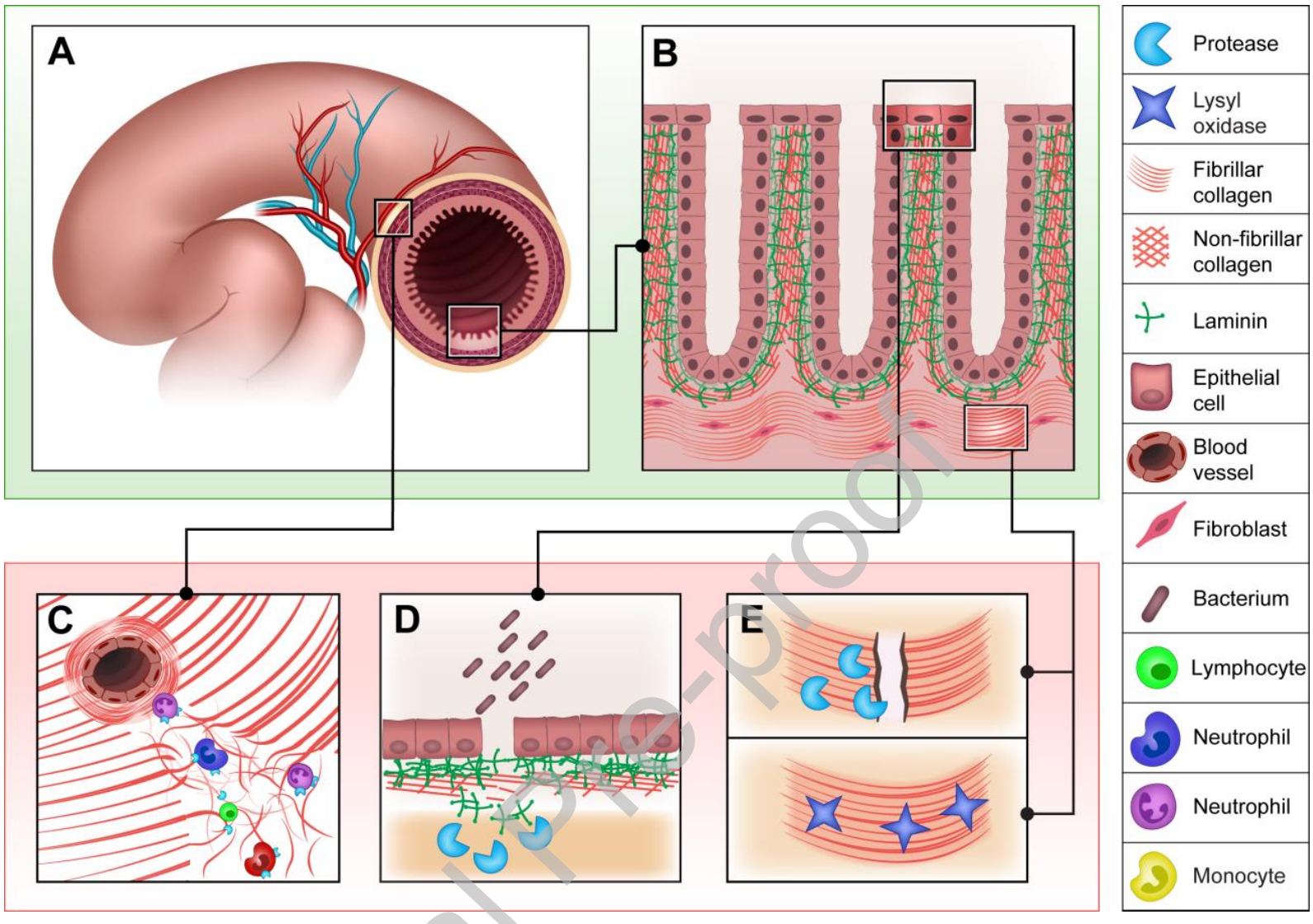

